\title{
Glycogen synthase kinases $3 \alpha$ and $3 \beta$ in cardiac myocytes: regulation and consequences of their inhibition
}

Article

Accepted Version

Markou, T., Cullingford, T. E., Giraldo Ramirez, A., Weiss, S. C., Alsafi, A., Fuller, S. J., Clerk, A. and Sugden, P. H. (2008) Glycogen synthase kinases $3 \alpha$ and $3 \beta$ in cardiac myocytes: regulation and consequences of their inhibition. Cellular Signalling, 20 (1). pp. 206-218. ISSN 0898-6568 doi: https://doi.org/10.1016/j.cellsig.2007.10.004 Available at https://centaur.reading.ac.uk/18419/

It is advisable to refer to the publisher's version if you intend to cite from the work. See Guidance on citing.

To link to this article DOI: http://dx.doi.org/10.1016/j.cellsig.2007.10.004

Publisher: Elsevier

All outputs in CentAUR are protected by Intellectual Property Rights law, including copyright law. Copyright and IPR is retained by the creators or other copyright holders. Terms and conditions for use of this material are defined in the End User Agreement. 


\section{CentAUR}

Central Archive at the University of Reading

Reading's research outputs online 
Glycogen synthase kinases $3 "$ and $3 \$$ in cardiac myocytes: regulation and consequences of their inhibition

Thomais Markou, Timothy E. Cullingford, Alejandro Giraldo, Sophie C. Weiss, Anwar Ali Amin, Stephen J. Fuller, Angela Clerk, Peter H. Sugden*

National Heart and Lung Institute (NHLI) Division, Faculty of Medicine, Imperial College London, Flowers Building, Armstrong Road, London SW7 2AZ, UK

Running title: Glycogen synthase kinase 3 in cardiac myocytes

Address for correspondence related to the submission process: Peter H. Sugden, NHLI Division, Faculty of Medicine, Imperial College London, Flowers Building (4th Floor), Armstrong Road, London SW7 2AZ, UK. Tel.: 44-20-7594-3410; Fax: 44-20-7594-3419; Email: p.sugden@imperial.ac.uk.

* Corresponding author. Tel.: +44 207594 3410; fax: +44 207594 3419. E-mail address: p.sugden@imperial.ac.uk (P.H. Sugden). 


\section{Abstract}

Inhibition of glycogen synthase kinase $3 \$(G S K 3 \$)$ as a consequence of its phosphorylation by protein kinase B/Akt (PKB/Akt) has been implicated in cardiac myocyte hypertrophy in response to endothelin-1 or phenylephrine. We examined the regulation of GSK3" (which we show to constitute a significant proportion of the myocyte GSK3 pool) and GSK3\$ in cardiac myocytes. Although endothelin increases phosphorylation of GSK3 and decreases its activity, the response is less than that induced by insulin (which does not promote cardiac myocyte hypertrophy). GSK3 phosphorylation induced by endothelin requires signalling through the extracellular signal-regulated kinase 1/2 (ERK1/2) cascade and not the PKB/Akt pathway, whereas the reverse is true for insulin. Cardiac myocyte hypertrophy involves changes in morphology, and in gene and protein expression. The potent GSK3 inhibitor 1azakenpaullone increases myocyte area as a consequence of increased cell length whereas phenylephrine increases both length and width. Azakenpaullone or insulin promotes AP1 transcription factor binding to an AP1 consensus oligonucleotide, but this was significantly less than that induced by endothelin and derived principally from increased binding of JunB protein, the expression of which was increased. Azakenpaullone promotes significant changes in gene expression (assessed by Affymetrix microarrays), but the overall response is less than with endothelin and there is little overlap between the genes identified. Thus, although GSK3 may contribute to cardiac myocyte hypertrophy in some respects (and presumably plays an important role in myocyte metabolism), it does not appear to contribute as significantly to the response induced by endothelin as has been maintained.

Keywords: Glycogen synthase kinase 3; cardiac myocytes; endothelin-1; insulin; hypertrophy. 


\section{Introduction}

Glycogen synthase kinase 3 (GSK3) was originally identified as a Ser-/Thr-protein kinase which phosphorylates and inhibits glycogen synthase [1]. In the preprandial or fasted state, GSK3 is catalytically-active and glycogenolysis predominates. On feeding, the insulinstimulated phosphorylation and consequent inhibition of GSK3 assists in promoting glycogen synthesis and accumulation [2]. In this case, inhibition of GSK3 is mediated by the signalling pathway which involves phosphoinositide 3-kinase (PI3K), 3-phosphoinositidedependent kinase 1 (PDK1) and protein kinase B/Akt (PKB/Akt). However, it is now recognised that GSK3 plays a much wider role in biological processes [3-7]. For example, it is central to the developmentally-important Wnt/Frizzled/\$-catenin pathway and this function appears to be largely separate from its role in the regulation of glycogen metabolism [8-10].

Mammalian cells express two isoforms of GSK3 which exhibit a high degree of sequence similarity in their catalytic domains, GSK3" (molecular mass $51 \mathrm{kDa}$ ) and GSK3\$ (molecular mass $47 \mathrm{kDa}$ ). GSK3 is phosphorylated on at least two residues. There are 'facilitative' autophosphorylations of GSK3" (Tyr-279) and GSK3\$(Tyr-216) required for GSK3 activity [11-13], and there are regulatory phosphorylations of GSK3" (Ser-21) and GSK3\$(Ser-9) which inhibit its kinase activity [3-7]. PKB/Akt phosphorylates GSK3" (Ser21) and GSK3\$(Ser-9), and these residues lie in a typical PKB/Akt consensus substrate motif [14]. However, GSK3" (Ser-21)/GSK3\$(Ser-9) are phosphorylated by other kinases which also recognise the "PKB/Akt consensus sequence". These include particularly the p70/p85ribosomal protein S6 kinases (S6K1 and S6K2) and the p90-ribosomal protein S6 kinases (RSK1-4) $[4,5,7,15]$. The active form of GSK3 phosphorylates its substrates in a 'relay' fashion which requires an initial 'priming' phosphorylation at a Ser-/Thr-residue lying four 
residues C-terminal (+4) to the Ser-/Thr- phosphorylated by GSK3, and this 'priming' phosphorylation is catalysed by one of several protein kinases [7]. The $\operatorname{Ser}(P) / \operatorname{Thr}(P)$ initially phosphorylated by GSK3 can serve subsequently as a priming phosphorylation for any Ser-/Thr-residue lying in the -4 position. These relays can be extended. For example, four GSK3 sites and a priming site are present between residues 641-657 in Homo sapiens muscle glycogen synthase.

GSK3 is important in Wnt-dependent cardiac development and growth [16]. However, it has also been implicated in adaptive growth of the postnatal heart [17-21]. The mammalian cardiac myocyte withdraws from the cell cycle in the perinatal period but expands in size during the maturational growth of the organism. In addition to maturational growth, cardiac myocytes can undergo adaptive, hypertrophic growth in vivo or ex vivo in response to an increase in cardiac workload [22,23], or to stimulation of a variety of transmembrane receptors (e.g. the endothelin $\mathrm{ET}_{\mathrm{A}}$ receptor or the " ${ }_{1}$-adrenergic receptor) [24-27]. In vivo, myocardial hypertrophy (to which the expanded cardiac myocytes contribute) is a major adaptive response which allows the heart to accommodate any physiological or pathological increase in loading that it encounters. However, in chronic pathological overloading (e.g. in hypertension), this adaptive phase may ultimately degrade into the maladaptive state of heart failure $[23,28]$. One proposal is that catalytically-active GSK3 inhibits the development of hypertrophy in the unstimulated myocyte, and inhibition of GSK3 (particularly GSK3\$) through PI3K-dependent signalling leads to hypertrophy [17-21]. Although GSK3 may participate in the cardiac myocyte hypertrophy induced by Gq-coupled protein receptor (GqPCR) agonists such as endothelin-1 (ET-1) acting at the $\mathrm{ET}_{\mathrm{A}}$ receptor and adrenergic receptor agonists $[17,18]$, it is by no means the only signalling protein implicated in the response. Others proteins/pathways include the extracellular signal- 
regulated kinase 1 and 2 (ERK1/2) cascade, other mitogen-activated protein kinase cascades, and $\mathrm{Ca}^{2+}$-dependent signalling pathways [29-34]. Furthermore, ERK1/2 phosphorylate and activate RSKs [35], for which GSK3 is a known substrate [15]. Here, we demonstrate differential signalling to GSK3 from ET-1 and insulin, and we characterise the morphological and transcriptional changes induced in cardiac myocytes by inhibiting GSK3 with a small molecule inhibitor, 1-azakenpaullone [36].

\section{Materials and methods}

\subsection{Materials}

Polyclonal antibodies to GSK3" and phospho-GSK3" (Ser-21)/phospho-GSK3\$(Ser9), and the monoclonal antibody to GSK3\$ were from Cell Signaling Technology (catalogue nos. 9338, 9331 and 9315, respectively). The monoclonal antibody to phospho-GSK3\$(Tyr216), which also cross-reacts with phospho-GSK3" (Ser-279), was from BD Transduction Laboratories (Pharmingen) (catalogue no. 612312). The monoclonal antibody to cardiac troponin T was from Stratech Scientific (catalogue no MS-295-P1). Secondary/tertiary antibodies and fluorescent mounting medium were from Dako. Antibodies to AP-1 components were from Santa Cruz Biotechnology Inc. as follows: c-Jun, sc-45X; JunB, sc46X; JunD, sc-74X; c-Fos, sc-52X; FosB, sc-48X; Fra1, sc-605X; and Fra2, sc-171X. [( $\left.{ }^{32} \mathrm{P}\right] \mathrm{ATP}$, streptavidin-Texas Red, rainbow molecular weight markers, and Hyperfilm MP were from GE Healthcare. ET-1 was from Bachem. Human Actrapid insulin was from Nova Nordisk Pharmaceutical Limited. PMA, Hoescht 33258 and other biochemicals were from 
Sigma-Aldrich Chemical Co. LY294002 and U0126 were from Alexis. 1-Azakenpaullone was from Calbiochem.

2.2. Primary culture of neonatal rat cardiac myocytes and preparation of whole cell extracts

Myocytes were dissociated from the ventricles of 1- to 3-day-old Sprague-Dawley rat hearts by an adaptation of the method of Iwaki et al. [26] as previously described [37]. Unless stated otherwise, cells were plated at a density of $4 \times 10^{6}$ cells/60 mm Primaria culture dish (BD Biosciences) for $18 \mathrm{~h}$ in 15\% (v/v) foetal calf serum, then serum was withdrawn for the $24 \mathrm{~h}$ before experimentation. Myocytes were exposed to ET-1 (100 nM), PMA (1 : M) or insulin (50 mU/ml, $300 \mathrm{nM}$ ) with or without pre-treatment (for $10 \mathrm{~min}$ ) with inhibitors. Following exposure to agonists, myocytes were washed in ice-cold phosphate-buffered saline (PBS). For whole cell extracts, myocytes were scraped into in 150 : 1/dish of Buffer A [20 mM \$-glycerophosphate pH 7.5, 20 mM NaF, 2 mM EDTA, $0.2 \mathrm{mM} \mathrm{Na}_{3} \mathrm{VO}_{4}, 10 \mathrm{mM}$ benzamidine, 5 mM dithiotheitol (DTT), 300 : M phenylmethylsulphonyl fluoride (PMSF), 200 : M leupeptin, 2 : M microcystin LR, 10 : M trans-epoxy-succinyl-L-leucylamido-(4guanidino)-butane (E64), containing $1 \%$ (v/v) Triton X-100]. Samples were incubated on ice for $10 \mathrm{~min}$ and then centrifuged $\left(10,000 \times \mathrm{g}, 5 \mathrm{~min}, 4^{\circ} \mathrm{C}\right)$. The supernatants were retained.

\subsection{Preparation of nuclear extracts}

Nuclear extracts were prepared as previously described [38]. Cardiac myocytes ( $4 \times$ $10^{6}$ cells/60 mm culture dish) were washed in ice-cold PBS and scraped into $150: 1$ ice-cold 
Buffer B (10 mM HEPES pH 7.9, $10 \mathrm{mM} \mathrm{KCl,} 1.5 \mathrm{mM} \mathrm{MgCl}_{2}, 0.3 \mathrm{mM} \mathrm{Na} \mathrm{VO}_{4}, 5 \mathrm{mM}$ DTT, 300 : M PMSF, 200 : M leupeptin, 2 : M microcystin LR, 10 : M E64). Samples were centrifuged $\left(10,000 \times \mathrm{g}, 5 \mathrm{~min}, 4^{\circ} \mathrm{C}\right)$ and the pellets washed in $100: 1$ Buffer B containing 0.1\% (v/v) Nonidet P40. Samples were centrifuged (10,000 × g, $5 \mathrm{~min}, 4 / \mathrm{C})$ and the pellets resuspended in 50 : l Buffer C [20 mM HEPES pH 7.9, $420 \mathrm{mM} \mathrm{NaCl}, 1.5 \mathrm{mM} \mathrm{MgCl}, 0.3$ mM Na $\mathrm{VO}_{4}, 0.2$ mM EDTA, 25\% (v/v) glycerol, 5 mM DTT, 300 : M PMSF, 200 : M leupeptin, 2 : M microcystin LR, 10 : M E64]. Suspensions were incubated on ice (1 h), then centrifuged $(10,000 \times \mathrm{g}, 5 \mathrm{~min}, 4 / \mathrm{C})$ and supernatants transferred to Eppendorf tubes. Protein concentrations were determined by the Bradford method [39]. Extracts were snapfrozen in liquid $\mathrm{N}_{2}$ and stored at $-80 / \mathrm{C}$.

\subsection{Immunoblot analysis}

Samples were boiled with 0.33 vol of SDS-polyacrylamide gel electrophoresis sample buffer [10\% SDS (w/v), 13\% glycerol (v/v), 300 mM Tris-HCl, pH 6.8, 130 mM DTT, 0.2\% bromophenol blue (w/v)]. Proteins [20 : g for total or nuclear extracts, $25: 1$ for Fast Protein Liquid Chromatography (FPLC) fractions] were separated by SDS-polyacrylamide gel electrophoresis using $10 \%(\mathrm{w} / \mathrm{v})$ resolving gels and $6 \%(\mathrm{w} / \mathrm{v})$ stacking gels, and transferred to nitrocellulose as described previously [40]. Non-specific binding sites were blocked with 5\% (w/v) non-fat milk powder in TBST [20 mM Tris-HCl pH 7.5, 137 mM NaCl, 0.1\% (v/v)

Tween 20]. Membranes were incubated (overnight, $4^{\circ} \mathrm{C}$ ) with primary antibodies diluted in TBST containing $1 \%(\mathrm{w} / \mathrm{v})$ bovine serum albumin. Primary antibodies were used at the following dilutions: GSK3", GSK3\$ and phospho-GSK3" (Ser-21)/phospho-GSK3\$(Ser-9), 1/1000; phospho-GSK3\$(Tyr-216), 1/2000 for total extracts and 1/1000 for fractions from 
FPLC; JunB, 1/2000. Antibodies to GSK3" and GSK3\$ were mixed when simultaneous detection was required. Membranes were washed in TBST, incubated (60 min, room temperature) with horseradish peroxidase- (HRP-) conjugated secondary antibodies (1/5000) in TBST containing 1\% (w/v) nonfat milk powder, and were then washed in TBST. For FPLC fractions immunoblotted for GSK3", GSK3\$ or phospho-GSK3" (Ser-21)/phosphoGSK3\$(Ser-9), an amplification step with goat anti-rabbit/mouse immunoglobulin secondary antibodies [1/5000 in TBST containing 1\% (w/v) non-fat milk powder] was included, followed by incubation with HRP-conjugated anti-goat immunoglobulin tertiary antibodies as described above. Bands were detected by enhanced chemiluminescence (Santa Cruz Biotechnology Inc.) using Hyperfilm MP.

\subsection{Fast protein liquid chromatography (FPLC)}

Myocytes $\left(16 \times 10^{6}\right)$ were scraped into Buffer D [25 mM HEPES pH 7.1, 2 mM EDTA, 2 mM EGTP, $0.1 \%$ (v/v) 2-mercaptoethanol, 5\% (v/v) glycerol, 0.06\% Brij 35, 0.3 $\mathrm{mM} \mathrm{Na} \mathrm{VO}_{4} 1 \mathrm{mM}$ benzamidine] containing 1\% Triton X100, $0.1 \mathrm{M} \mathrm{NaCl}, 300$ : M PMSF, 200 : M leupeptin, 2 : M microcystin LR, and 10 : M E64. Samples were centrifuged $(10,000 \times \mathrm{g}, 10 \mathrm{~min}, 4 / \mathrm{C})$ and the supernatants were re-centrifuged. Clarified supernatants were applied to a MonoS HR5/5 column equilibrated with Buffer D containing $0.1 \mathrm{M} \mathrm{NaCl}$ (preliminary screening experiments revealed that no GSK3 activity eluted below $0.1 \mathrm{M}$ $\mathrm{NaCl}$ ). The column was washed with Buffer D containing $0.1 \mathrm{M} \mathrm{NaCl}$ (5 ml) and proteins were eluted using a linear $\mathrm{NaCl}$ gradient $(0.1-0.3 \mathrm{M} \mathrm{NaCl}, 10 \mathrm{ml})$ at a flow rate of $1 \mathrm{ml} / \mathrm{min}$ with collection of $0.5 \mathrm{ml}$ fractions. Samples from each fraction were assayed for GSK3 
activity or boiled with $0.33 \mathrm{vol}$ of SDS-polyacrylamide gel electrophoresis sample buffer for immunoblotting

\subsection{Assay of GSK3 activity}

GSK3 activity in FPLC fractions was assayed by the incorporation of [ $\left(-{ }^{32} \mathrm{P}\right] \mathrm{ATP}$ into a 'primed' phosphopeptide substrate (YRRAAVPPSPSLSRHSSPHQ(pS)EDEEE [41], synthesised by the Advanced Biotechnology Centre, Imperial College London) in the absence or presence of 1-azakenpaullone (10: M) to inhibit GSK3. Column fractions (40: l) were added to 5 : 1 of Buffer E [50 mM Tris-HCl pH 7.5, 0.1 \% (v/v) 2-mercaptoethanol, 100 : M 'primed’ phosphopeptide substrate [41], with 100 : M 1-azakenpaullone when required]. Reactions were initiated by addition of $5: 11 \mathrm{mM}$ ATP/100 $\mathrm{mM} \mathrm{MgCl}{ }_{2}$ containing 2 : Ci $\left[\left({ }^{32} \mathrm{P}\right] \mathrm{ATP}\right.$ and incubations were for $20 \mathrm{~min}$ at $30^{\circ} \mathrm{C}$. Assays were terminated by spotting 40 : l of the reaction mixture onto Whatman P81 papers which were then washed in $75 \mathrm{mM}$ $\mathrm{H}_{3} \mathrm{PO}_{4}$ (4 × $\left.15 \mathrm{~min}\right)$. Radioactivity incorporated was determined by „erenkov counting. GSK3 activity was taken as the difference in ${ }^{32} \mathrm{P}$ incorporation between assay in the absence and presence of 1-azakenpaullone.

\subsection{Immunostaining and planimetry}

Glass coverslips were placed in Primaria 35 mm culture dishes and coated with gelatin $[1 \%(\mathrm{v} / \mathrm{v})]$ then laminin (20: $\mathrm{g} / \mathrm{ml}$ in PBS, $2 \mathrm{~h}$, room temperature). Dishes plus coverslips were washed three times with PBS. Myocytes were plated at $1.5 \times 10^{6}$ cells/dish in serum (18 h). Serum was withdrawn (24 h) and myocytes were exposed to $100: \mathrm{M}$ phenylephrine, 10 : M 1-azakenpaullone, or $10 \mathrm{mM} \mathrm{LiCl} \mathrm{(24} \mathrm{h).} \mathrm{Cells} \mathrm{were} \mathrm{washed} \mathrm{three}$ 
times with PBS and fixed in 4\% (v/v) formaldehyde (10 min, room temperature). Following permeabilisation with $0.3 \%(\mathrm{v} / \mathrm{v})$ Triton X-100 (10 min, room temperature), non-specific binding was blocked with $2.5 \%(\mathrm{w} / \mathrm{v})$ bovine serum albumin in 0.3\% (v/v) Triton X-100 (10 min, room temperature). Antibodies and fluorophores were diluted in PBS, all incubations were at room temperature in a humidified chamber, and coverslips were washed three times in PBS after each stage of the immunostaining procedure. Myocytes were stained with mouse monoclonal primary antibodies to phospho-GSK3" (Tyr-279)/phospho-GSK3\$(Tyr216) (1/100, $60 \mathrm{~min})$, with biotinylated anti-mouse immunoglobulin secondary antibodies (1:200, $60 \mathrm{~min})$ and streptavidin-Texas Red (1:200, $30 \mathrm{~min})$. Nuclei were stained with Hoechst 33258 (50 : g/ml, 15 min). Myocytes were stained or additionally counterstained with mouse monoclonal primary antibodies to troponin $\mathrm{T}(1 / 40,60 \mathrm{~min})$ with FITC-conjugated anti-mouse immunoglobulin secondary antibodies (1/200, $60 \mathrm{~min})$. Coverslips were mounted using fluorescent mounting medium and viewed by fluorescence microscopy. Cardiac myocyte area was measured by digital planimetry (Scion Image Alpha 4.03, Scion Corporation, USA). At least 100 myocytes were measured for a single experiment and 4 independent experiments were conducted. Maximum cell length and width were also measured. Results were normalised to the mean value for the control cells for each experiment and results are presented as the means \pm S.E.M. for the 4 independent observations.

\subsection{Electrophoretic mobility shift assays (EMSAs)}

An oligonucleotide probe containing the consensus AP1 sequence (5'-

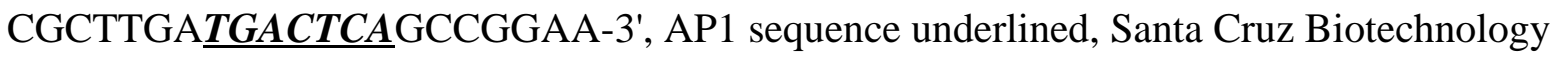


Inc.) was 5'-radiolabelled (30 min, 37/C) in 12 : l containing 30 ng double-stranded oligonucleotide, 10 units T4 polynucleotide kinase (Promega), 15 : Ci [( $\left.-^{32} \mathrm{P}\right] \mathrm{ATP}$ and 1.2 : 1 of commercial $10 \times \mathrm{T} 4$ kinase buffer (Promega). Unincorporated $\left[\left(-{ }^{32} \mathrm{P}\right] \mathrm{ATP}\right.$ was removed by centrifugation $(700 \times \mathrm{g}$, $3 \mathrm{~min}$, room temperature) through pore size 10 Chromaspin columns (BD Biosciences - Clontech) in $10 \mathrm{mM}$ Tris-HCl pH 8.0, 1 mM EDTA. The probe was diluted to a working concentration of $0.4 \mathrm{ng} /$ : 1 .

Cardiac myocyte nuclear extracts (10 : g protein) were incubated (10 min, room temperature) with 4 : l Buffer F [50 mM Tris-HCl pH 7.5, 250 mM NaCl, 5 mM EDTA, 1 mM DTT, 25\% (v/v) glycerol] and $1:$ g poly dI-dC in a total volume of $20: 1$. Radiolabelled probe (0.4 ng) was added and the mixture incubated (20 min, room temperature). DNAprotein complexes were separated by electrophoresis $(120 \mathrm{~V}, 3 \mathrm{~h})$ non-denaturing polyacrylamide gels [5\% (w/v) acrylamide, $0.166 \%(\mathrm{w} / \mathrm{v})$ bisacrylamide, $0.05 \%(\mathrm{w} / \mathrm{v})$ ammonium persulphate, 0.1\% (v/v) TEMED in Buffer G (45 mM Tris base, $45 \mathrm{mM} \mathrm{H}_{3} \mathrm{BO}_{4}, 1$ mM EDTA)] with Buffer $\mathrm{G}$ as running buffer. Gels were dried (80/C, $45 \mathrm{~min}$ ) onto Whatman 3MM paper and exposed to Hyperfilm MP for 24 - 48 h at -80/C. For supershift assays, nuclear extracts ( $3:$ g protein) were pre-incubated $(1 \mathrm{~h}, 4 / \mathrm{C})$ with $1-2:$ g of primary antibody and EMSAs then performed as described.

\subsection{RNA preparation and microarray analysis.}

Myocytes ( $4 \times 10^{6}$ cells/condition) were unstimulated, or were exposed to $10: \mathrm{M}$ 1-azakenpaullone or $100 \mathrm{nM}$ ET-1 for $60 \mathrm{~min}$. Total RNA was extracted, and cRNA synthesised from $10:$ g total RNA and purified as described [42]. To minimise the effects of variation between separate myocyte preparations, equal amounts of RNA were pooled from 3 independent experiments prior to cRNA synthesis. Three separate experiments were 
performed for each condition (i.e. 9 myocyte preparations in total). Fragmentation of antisense cRNA and hybridisation to Affymetrix rat genome 2302.0 arrays were performed at the CSC Microarray Centre (Hammersmith Campus, Imperial College London) according to their standard protocols (microarray.csc.mrc.ac.uk). The MIAME-compliant data were exported to ArrayExpress (E-MIMR-681). Preliminary analysis of hybridisation data was with Affymetrix GeneChip Operating System, GCOS. The data were imported into GeneSpring GX 7.3.1 (Agilent Technologies) as tab-delimited text files. $\log _{10}$ values were used for analysis with values set to a minimum of 0.01 and, for each data-set, the data were normalised per array (to the 50th percentile) and values for each probe set were normalised to the corresponding controls (prepared and hybridised simultaneously). A confidence filter was applied and genes were selected if present or marginal in all controls, all samples from myocytes exposed to 1-azakenpaullone or all samples from myocytes exposed to ET-1. One-way non-parametric t-tests were performed for each transcript for each condition relative to the appropriate controls (GeneSpring GX). The false discovery rate was set to $<0.05$ and multiple testing correction performed using the Benjamini and Hochberg false discovery rate algorithm. Transcripts were filtered on the basis of $>2$-fold difference. All genes were confirmed by BLAST search and were correct as of August 2007.

\subsection{Quantitative PCR ( $q P C R)$}

RNA was extracted and cDNA synthesized using reverse transcription as previously described [42]. qPCR was performed using a Real-Time PCR System model 7500 (Applied Biosystems). Amplifications were carried out in optical 96-well reaction-plates (Applied Biosystems) with each well containing 12.5 : l of SYBR Green Jump Start Taq Readymix (Sigma Aldrich Chemical Co.), 5 : l of oligonucleotide primers (5 pmol each of forward and 
reverse primers) and $7.5: 1$ of cDNA template (diluted 15-fold in water). Primers used were as follows: prostaglandin-endoperoxide synthase 2 (Ptgs2, also known as cyclooxygenase 2, accession no. NM_017232), forward primer: 5'-CAAGACAGATCAGAAGCGAG-3' (712-731), reverse primer: 3'-GTCTGTCTAGAGTTTCACCG-5' (784-803), 92 bp product; cyclin G2 (Ccng2_predicted, accession no. XM_223270), forward primer: 5'ACTGCACTATGAGCTGGAAG-3' (558-577), reverse primer: 3'ATCGAGGCTCAGAACTTCCT-5' (644-663), 106 bp product; adrenomedullin (accession no. NM_012715), forward primer: 5'-GCTCGTTGATGAGAAGACAG-3' (336-355), reverse primer: 3'-GGTAGCGTTTGACTCGAATG-5' (435-454), 119 bp product; glyceraldehyde 3phosphate dehydrogenase (Gapd, accession no. NM_017008), forward primer: 5'GCTGGCATTGCTCTCAATGACA-3' (1738-1759), reverse primer: 3'TCCACCACCCTGTTGCTGTA-5' (1801-1820), 83 bp product. qPCR analysis of Gapd was performed as a control and the relative quantification protocol was used. PCR conditions for all primer pairs were 50/C for $2 \mathrm{~min}$, 95/C for $10 \mathrm{~min}$ (Jump-Start Taq polymerase activation step), followed by 40 cycles of 95/C for $15 \mathrm{~s}$ and 59/C for $60 \mathrm{~s}$. Following qPCR, dissociation curve analysis was routinely performed to check for aberrant amplification products (e.g. primer-dimers).

\subsection{Densitometry and statistical analysis}

For immunoblots and EMSA autoradiographs, bands were quantified by scanning densitometry using Imagemaster 1D calibrated with Kodak photographic step tablet no. 2

(GE Healthcare). Statistical analysis was performed using GraphPad Prism. One-way ANOVA (repeated measures where appropriate) was used with a multiple comparison test (Tukey for full multiple testing; Dunnett for testing against controls only). 


\section{Results}

\subsection{Stimulation of phosphorylation of GSK3 "(Ser-21) and GSK3 \$(Ser-9) in cardiac}

myocytes by ET-1, PMA or insulin

Immunoblot analysis demonstrated that both GSK3" and GSK3\$ are expressed in cardiac myocytes (Fig. 1A-C, lower panels). Using phosphorylation site-specific antibodies, we showed that maximally-effective concentrations (data not shown) of ET-1 (100 nM), PMA (1 : M) or insulin (300 nM) promoted rapid increases (maximal in 2-5 min) in the phosphorylation of GSK3" (Ser-21) and GSK3\$(Ser-9) (Fig. 1A-C, upper panels; quantification in Fig. 1D and E). Phosphorylation remained relatively constant over the 5 30 min period. The relative increases in phosphorylation of GSK3" (Ser-21) and GSK3\$(Ser-9) compared with zero-time controls were apparently greater for insulin than for ET-1 or PMA. A direct comparison of the effects of the three agonists (20 min) confirmed this to be the case (Fig. 1F, quantification in Fig. 1G).

GSK3" (Ser-21)/GSK3\$(Ser-9) may be phosphorylated by a variety of protein kinases lying downstream from either PI3K (i.e. PKB/Akt) or the ERK1/2 cascade (e.g. RSKs) $[4,5,7]$. U0126 and LY294002 are widely used as inhibitors of the ERK1/2 cascade [43] and of PI3K [44], respectively. U0126 reduced the phosphorylation of GSK3" (Ser-21) and GSK3\$(Ser-9) stimulated by ET-1 (Fig. 2A, D and E) or PMA (Fig. 2B, D and E), whereas LY294002 was largely ineffective. Conversely, LY294002 reduced the phosphorylation of GSK3" (Ser-21) and GSK3\$(Ser-9) stimulated by insulin (Fig. 2C, D and E), whereas U0126 was largely ineffective. Thus, ET-1 and PMA induce phosphorylation of 
GSK3" (Ser-21) and GSK3\$(Ser-9) primarily through the ERK1/2 cascade, whereas insulin induces phosphorylation primarily through PI3K.

\subsection{Phosphorylation of GSK3 "(Tyr-279) and GSK3 \$(Tyr-216)}

Phosphorylation of GSK3" (Tyr-279) and GSK3\$(Tyr-216) is essential for their catalytic activity. Although there have been reports that agonists cause dephosphorylation or phosphorylation of these residues and this represents a means whereby GSK3 can be inhibited or activated in intact cells [45-48], this is not necessarily the case $[7,13,49]$. We examined the phosphorylation of GSK3" (Tyr-279) and GSK3\$(Tyr-216) in cardiac myocytes. The monoclonal antibody to phospho-GSK3\$(Tyr-216) cross-reacts with phospho-GSK3" (Ser-279), presumably because the amino acid sequences of residues 190305 in rat GSK3\$ (accession no. NP_114469) and residues 253-368 in rat GSK3" (accession no. NP_059040) are identical. We detected phospho-GSK3" (Tyr-279) and phosphoGSK3\$(Tyr-216) in approximately equal apparent abundances (Fig. 3A-C). Assuming that the affinity of the monoclonal antibody for both GSK3 isoforms is similar (because of the sequence identity) and that GSK3" (Tyr-279) and GSK3\$(Tyr-216) are stoicheiometrically phosphorylated [13], this suggests that GSK3" contributes significantly to the GSK3 complement in cardiac myocytes and cannot be ignored. However, there were not any changes in the phosphorylation of GSK3" (Tyr-279) or GSK3\$(Tyr-216) in cardiac myocytes treated with ET-1 (Fig. 3A), PMA (Fig. 3B) or insulin (Fig. 3C) over 30 min.

The phosphorylation of GSK3" (Tyr-279) and GSK3\$(Tyr-216) probably represents autophosphorylation. Chemical inhibition of GSK3 leads to dephosphorylation of GSK3" (Tyr-279) and GSK3\$(Tyr-216) in other cells [7,13]. In cardiac myocytes exposed to 
the GSK3 inhibitor, 1-azakenpaullone (10: M) [36], the total pool of GSK3\$ did not change, but phospho-GSK3" (Tyr-279) and phospho-GSK3\$(Tyr-216) declined over a $12 \mathrm{~h}$ period (Fig. 3D and E). Phospho-GSK3" (Tyr-279) was apparently more rapidly dephosphorylated than phospho-GSK3\$(Tyr-216). These data are consistent with the view that phosphorylation of GSK3" (Tyr-279) and GSK3\$(Tyr-216) represents an autophosphorylation $[7,13]$. However, we do not know the mechanism of the dephosphorylation, nor do we understand its differential nature for the two GSK3 isoforms. Dephosphorylation (Fig. 3D and E) could represent a genuine dephosphorylation as a result of protein Tyr-phosphatase activity. Alternatively, turnover of phospho-GSK3" (Tyr-279) and phospho-GSK3\$(Tyr-216) proteins through protein degradation and resynthesis of GSK3 in the absence of autophosphorylation would lead to apparent dephosphorylation. In this regard, Cole et al. [13] have presented data with protein synthesis inhibitors suggesting that the dephosphorylation reflects a genuine phosphatase activity rather than protein turnover.

\subsection{Fast Protein Liquid Chromatography separation of GSK3}

GSK3" /GSK\$ were partially purified by MonoS FPLC of cardiac myocyte extracts from unstimulated cells and from cells exposed to ET-1 or insulin. Column fractions were assayed GSK3 activity and immunoblotted for phospho-GSK3" (Tyr-279)/phosphoGSK3\$(Tyr-216), phospho-GSK3" (Ser-21)/phospho-GSK3\$(Ser-9) and total GSK3\$ (Fig. 4). GSK3 activity eluted as a broad peak between $0.11-0.22 \mathrm{M} \mathrm{NaCl}$ (fractions 11-20) with a small shoulder (particularly apparent using control extracts) in fractions 13-14 (Fig. 4A). Total GSK3 activities (obtained by integrating areas under the peaks) were reduced by $54.2 \pm$ 
1.4\% with insulin and $31.9 \pm 6.4 \%$ with ET-1 (Fig. 4B). Thus, both insulin and ET-1 inhibit GSK3, but perhaps not to the extent that might have been anticipated.

Antibodies to phospho-GSK3" (Tyr-279)/phospho-GSK3\$(Tyr-216) proved effective for the detection of total GSK3 (i.e. both isoforms) by immunoblotting (Fig. 4C) and were used routinely for this purpose. Although total GSK3\$ antibodies were satisfactory for detecting protein in FPLC fractions, total GSK3" antibodies did not detect any bands. Phospho-GSK3" (Tyr-279) eluted predominantly in fractions 12-17, with phosphoGSK3\$(Tyr-216) eluting in fractions 14-20 (Fig. 4C). GSK3\$ immunoreactivity reflected phospho-GSK3\$(Tyr-216) immunoreactivity (Fig. 4D). Thus, GSK3\$ probably constitutes the majority of GSK3 activity in fractions 15-19 (Fig. 4A), and may indeed constitute the majority of GSK3 activity overall. GSK3" probably accounts for the shoulder of GSK3 activity in fractions 13-14 (Fig. 4A). A significant proportion of phospho-GSK3" (Tyr279)/phospho-GSK3\$(Tyr-216) (or total GSK3\$) eluted in fractions 2-4 of the isocratic wash (Fig. 4C), but there was no detectable GSK3 activity (data not shown). This pool of GSK3 did not bind to MonoS columns even in the total absence of any $\mathrm{NaCl}$ in the extraction buffer (myocytes were routinely extracted in $0.1 \mathrm{M} \mathrm{NaCl}$ ). There were no gross changes in the elution profiles of phospho-GSK3" (Tyr-279) or phospho-GSK3\$(Tyr-216) (or, indeed, total GSK3\$) in cells treated with ET-1 or insulin.

The phospho-GSK3" (Ser-21)/phospho-GSK3\$(Ser-9) antibodies detected little immunoreactivity in FPLC fractions from control extracts, but immunoreactivity was readily detected in fractions 13-18 using extracts of myocytes exposed to ET-1 or insulin (Fig. 4E). We directly compared fractions obtained from control myocytes and myocytes exposed to ET-1 or insulin (Fig. 4F). ET-1 or insulin increased phospho-GSK3" (Ser-21)/phosphoGSK3\$(Ser-9) (relative to controls) in the fractions from the isocratic wash and in gradient 
fractions 16-18, with insulin having the greater effect. The increase in phospho-GSK3" (Ser21)/phospho-GSK3\$(Ser-9) immunoreactivity in fractions 2-3 (Fig. 4E and F) implies that phosphorylation of GSK3" (Ser-21) and GSK3\$(Ser-9) in this pool can be increased over control values. Thus, the absence of GSK3 activity in fractions 2-3 does not result from the fact that GSK3" (Ser-21) and GSK3\$(Ser-9) in these fractions are already fully phosphorylated.

\subsection{Subcellular localisation of GSK3 and the morphological effects of its inhibition in cardiac myocytes}

Subcellular localisation of GSK3 was assessed by immunostaining with antibodies to phospho-GSK3" (Tyr-279)/phospho-GSK3\$(Tyr-216) with counterstaining of nuclei by Hoechst 33258 (Fig. 5A and B). Myocyte identity was confirmed by troponin T immunostaining (data not shown). Phospho-GSK3" (Tyr-279)/phospho-GSK3\$(Tyr-216) immunostaining was strongest in myocyte nuclei, with considerably less in the cytoplasm (Fig. 5A). There were no gross changes in phospho-GSK3" (Tyr-279)/phospho-GSK3\$(Tyr216) localisation in cells exposed to ET-1 or insulin for 20 min (data not shown).

We compared the effects of inhibitors of GSK3 (1-azakenpaullone or LiCl) and the " adrenergic agonist, phenylephrine (a powerful hypertrophic agonist) on cardiac myocyte morphology. Phenylephrine promoted increased cardiac myocyte area (Fig. 5C and D). This was associated with increases in maximum cell length and width (Fig. 5E and F) such that the maximum length:maximum width ratio was increased to only a small extent compared with controls (Fig. 5G). Cardiac myocyte area was also increased by 1-azakenpaullone (Fig. 5C), but this primarily reflected increased cell length in the absence of any increase in cell width 
(Fig. 5E and F). Consequently, the maximum length:maximum width ratio was significantly increased (Fig. 5G). There was a small increase (42 $\pm 5 \%$ ) in the area of cells exposed to LiCl (Fig. 5D), but this did not reach statistical significance.

\subsection{Transcription factor binding to the AP1 consensus sequence}

The AP1 transcription factor complex is a homodimer of Jun family members (c-Jun, JunB and JunD) or a heterodimer of Jun family members and Fos family members (c-Fos, FosB, Fra1 and Fra2), though not all combinations are allowed [50,51]. GSK3 phosphorylates the C-terminal regions of Jun family transcription factors at sites proximal to their DNA binding domains, and this decreases their affinity for AP1 consensus sequences and their transactivating potential [52]. Conversely, inhibition of GSK3 and dephosphorylation of the GSK3 sites in the Jun family increases their transactivating potential. We examined the abilities of 1-azakenpaullone, insulin and ET-1 to increase binding of nuclear proteins to an AP1 oligonucleotide using EMSAs (Fig. 6A and B). ET-1 was the most effective agonist, but both 1-azakenpaullone and insulin were moderately effective (Fig. 6A and B). Antibodies to JunB clearly supershifted the bands after exposure of cells to each of the three agonists (Fig. 6C). Antibodies to other potential AP1 components produced a subshift (c-Fos) or reduced binding (for FosB, JunD or Fra2) suggesting that they may be involved, but there was no consistency between the three agonists.

Increased binding of JunB to AP1 sequences could reflect an increase in abundance or an increase in affinity caused by a reduction in phosphorylation of Ser-/Thr- residues proximal to the C-terminal DNA region. 1-Azakenpaullone (Fig. 6D) or insulin (Fig. 6E) 
increased the abundances of JunB within $1 \mathrm{~h}$, which may well explain the increase in binding to AP1 (Fig. 6A-C). By analogy with human JunB [52], rat JunB should contain GSK3 phosphorylation sites at Ser-248 and Thr-252, the priming phosphorylation being on Ser-256. We do not know whether there is a decrease in phosphorylation of these residues as well as an increase in abundance following inhibition of GSK3. The only available phosphopeptide antibody detects the priming phosphorylation (Ser-259 in Homo sapiens JunB). Not unexpectedly, we were unable to detect any changes in the phosphorylation state of this residue with 1-azakenpaullone, insulin or ET-1 (data not shown).

\subsection{Changes in gene expression}

Since GSK3 appears to be localised predominantly to the nucleus in cardiac myocytes and 1-azakenpaullone increases AP1 transcription factor binding to a consensus sequence, we examined the effects of 1-azakenpaullone $(1 \mathrm{~h})$ on global gene expression in cardiac myocytes using Affymetrix rat genome 2302.0 arrays. In response to 1-azakenpaullone, we identified significant changes in expression (FDR $<0.05$; 2-fold minimum change) in 26 established, protein-coding genes (Table 1). This contrasts with 127 established genes with significant changes in expression induced by ET-1 at $1 \mathrm{~h}$ (A. Clerk et al., manuscript submitted). Although expression of 12 genes was altered by 1-azakenpaullone or ET-1, only two were changed to a similar extent in response to either agonist. For 5 genes, the response to ET-1 was substantially greater than that induced by 1-azakenpaullone and a further 5 demonstrated opposing changes (i.e. 1-azakenpaullone promoted a decrease in expression whereas ET-1 increased expression). Overall, the effect of 1-azakenpaullone on gene expression did not resemble that induced by ET-1 suggesting that any effect of ET-1 on 
GSK3 activity does not necessarily influence changes in gene expression associated with hypertrophy. It is worth noting that 1-azakenpaullone did not increase the abundance of JunB mRNA above our nominal threshold value (2-fold). The increase in JunB protein induced by 1-azakenpaullone (Fig. 6D) may therefore reflect decreased degradation of JunB protein.

To validate the changes in gene expression induced by 1-azakenpaullone, we used qPCR to examine a more detailed time course for expression of prostaglandin-endoperoxide synthase 2 (Ptgs2, also known as cyclooxygenase 2), cyclin G2 (Ccng2) and adrenomedullin. Consistent with the microarray data (Table 1), expression of Ptgs2 mRNA was increased at 60 min (Fig. 7A). This increase was transient and expression declined almost to basal levels by 2 h. Ccng2 (Fig. 7B) and adrenomedullin (Fig. 7C) mRNAs were both decreased in expression with maximal decreases occurring at 60 - 90 min.

\section{Discussion}

In addition to participating in the Wnt/Frizzled pathway during cardiac development [16], inhibition of GSK3 has recently been implicated in the development of cardiac hypertrophy $[20,21]$. In cultured cardiac myocytes, \$-adrenergic stimulation induces aspects of the hypertrophic response in a manner dependent on the activation of PI3K and PKB/Akt, and the subsequent inhibition of GSK3\$ [17]. Similar conclusions have been drawn with ET-1 or phenylephrine as agonists, or by using chemical inhibition of GSK3 with LiCl [18]. These ex vivo observations were extended with the in vivo finding that raising left intraventricular pressure by transverse aortic constriction (TAC, a pro-hypertrophic intervention) inhibits GSK3 activity [18]. Furthermore, a transgenic mouse in which a 
constitutively-active (non-phosphorylatable) form of GSK3\$ [GSK3\$(Ser-9Ala)] is cardiospecifically expressed showed reduced hypertrophy in response to overexpression of constitutively-active calcineurin, infusion of isoprenaline or TAC [19]. There were no obvious effects of GSK3\$(Ser-9Ala) in unmanipulated control mice (although others have reported that cardiospecific overexpression of wild type GSK3\$ impairs post-natal growth of cardiac myocytes [53]). Thus, stimulation of the catalytic activity of GSK3 could represent an anti-hypertrophic intervention, possibly with therapeutic potential. Potentially contrasting with the proposed anti-hypertrophic action, a pro-apoptotic role of GSK3 activation has been identified in adult cardiac myocytes [48] and other cells [54]. Furthermore, cardiospecific overexpression of wild-type GSK3\$ causes contractile dysfunction, possibly as a result of disturbances in $\mathrm{Ca}^{2+}$ movements [53]. These experiments [48,53] suggest that activation of GSK3 might be deleterious. However, it is worth noting that, in vivo, replacement of the wild-type GSK3" and GSK3\$ genes with transgenes encoding constitutively-active GSK3" and GSK3\$ [GSK3" (Ser21Ala)/GSK3\$(Ser9Ala) 'knock-in' mice] does not appear to interfere with survival or baseline cardiac function [55,56]. In this situation, the fully-active mutant proteins in the 'knock-in' situation are expressed in the same abundances as the wildtype proteins in the wild-type animals, thus simulating the in vivo situation, rather than being overexpressed.

Here, we have extended the characterisation of GSK3 in the heart. Past reports have emphasised a predominant role for GSK3\$ (as opposed to GSK3") in cardiac myocyte hypertrophy. However, both isoforms are clearly expressed (Fig. 1A-C). Assuming that the phospho-GSK3\$(Tyr-216) antibody has similar affinity for phospho-GSK3" (Tyr-279) as for phospho-GSK3\$(Tyr-216) (see section 3.2), results shown in Fig. 3A-C suggest that GSK3" and GSK3\$ are present in approximately equal mass abundances in cardiac myocytes. In 
terms of activity under basal conditions, a combination of MonoS FPLC and immunoblotting with anti-phospho-GSK3\$(Tyr-216) suggests that GSK3\$ activity predominates over GSK3" activity by a factor of 5- to 10-fold (Fig. 4A and C). However, the catalytic activity of GSK3" is probably important in the heart. Unlike the situation in skeletal muscle where GSK3\$ activity seems the more important [55], GSK3" does participate significantly in the regulation of cardiac glycogen metabolism [56]. It is not known whether there is any GSK3 isoform specificity in terms of additional functional consequences in the heart. In whole animals and isolated cells, both similarities and differences between GSK3" and GSK3\$ have been detected [55-58]. We therefore believe that a role for both GSK3" and GSK3\$ particularly in myocyte hypertrophy should be considered.

Published studies emphasise the importance of PKB/Akt in the phosphorylation and inhibition of GSK3 by hypertrophic GPCR agonists [17,18,59]. However, GSK3 may be phosphorylated by other protein kinases. In cardiac myocytes, ET-1 and PMA rapidly and powerfully activate ERK1/2 [60] whereas they only weakly activate PKB/Akt [61,62]. Conversely, insulin is a powerful activator of $\mathrm{PKB} / \mathrm{Akt}$, but is ineffective in activating ERK1/2 [62,63]. Thus, the separate roles of these two signalling pathways can be partially delineated in cardiac myocytes by the use of these 'selective' agonists. Insulin at maximallyeffective concentrations causes the largest increase in phosphorylation of GSK3" (Ser21)/GSK3\$(Ser-9), with ET-1 or PMA being approximately half as effective (Fig. 1F and G) implying that PKB/Akt mediates insulin-stimulated phosphorylation of GSK3 and ERK1/2 mediate phosphorylation in response to ET-1 or PMA. Consistent with this, U0126 inhibits the phosphorylation of GSK3" (Ser-21) and GSK3\$(Ser-9) induced by ET-1 or PMA, but not insulin (Fig. 2). Conversely, LY294002 inhibits phosphorylation of GSK3" (Ser-21) and GSK3\$(Ser-9) induced by insulin, but not ET-1 or PMA (Fig. 2). Although potential 
problems relating to inhibitor specificity cannot be excluded [64,65], we conclude that the ERK1/2 cascade is primarily responsible for phosphorylation of GSK3 induced by ET-1 or PMA (possibly via the RSKs), whereas PI3K signalling is required for that induced by insulin (presumably via PKB/Akt). Our results for ET-1 contrast with those of Haq et al. [18] who concluded that phosphorylation of GSK3\$(Ser-9) involved activation of PI3K.

MonoS FPLC raises the possibility of separate pools of GSK3. Apart from the catalytically-active pool of GSK3 which eluted at $0.11-0.22 \mathrm{M} \mathrm{NaCl}$, we detected a pool of phospho-GSK3" (Tyr-279)/phospho-GSK3\$(Tyr-216) in the isocratic wash which was catalytically inactive (Fig. 4A, C and D). Although largely unphosphorylated on GSK3" (Ser-21)/GSK3\$(Ser-9) under control conditions, this pool became phosphorylated following exposure of the cells to insulin and, to a lesser extent, ET-1 (Fig. 4C and E) indicating that it is not inactive as a consequence of complete phosphorylation of the inhibitory Ser- residues. One explanation could be that this pool of GSK3 is inhibited by complexation with other proteins.

Catalytically-active GSK3 eluted from MonoS columns at $0.11-0.22 \mathrm{M} \mathrm{NaCl}$, and insulin or ET-1 increased phosphorylation GSK3" (Ser-21)/GSK3\$(Ser-9), partially inhibiting this GSK3 (Fig. 4A, E and F). Other studies used immunoprecipitation to purify GSK3 [41] for in vitro assays and similarly showed only partial inhibition of GSK3 in response to insulin or ET-1 [18]. However, such assays are known to be problematical in certain instances (K. Sakamoto, personal communication) and, although we could detect substantial GSK3\$ activity (only the anti-GSK3\$ antibody was suiyable for immunoprecipitation) in unstimulated cells using immunokinase assays, we were consistently unable to detect any inhibition by insulin or ET-1. This (and the partial rather than more extensive inhibition expected from our FPLC experiments) may relate to the mechanism by 
which phosphorylation of GSK3" (Ser-21)/GSK3\$(Ser-9) is inhibitory. These phosphorylations compete internally for the binding site of the priming phosphorylation on GSK3 substrates [71]. In an assay, therefore, an efficient phospho-peptide substrate such as that used here competes effectively for the same binding site. We suspect that the enzyme:substrate ratio is crucial in these assays.

The mechanisms involved in the anti-hypertrophic effects of (catalytically-active) GSK3 are not clear $[20,21]$ but presumably involve regulation of gene and protein expression. Changes in gene expression may result from degradation of the transcriptional co-regulator, \$-catenin [59,66,67] (as occurs in the Wnt/Frizzled pathway [8-10]), nuclear exclusion of pro-hypertrophic transcription factors such as the NFATs $[18,19]$ and GATA4 [68], and inhibition of the biological activity of the transcriptional co-regulator, myocardin [69]. At the level of translation, GSK3 may phosphorylate and reduce the biological activity of eukaryotic initiation factor 2B [70]. Immunostaining with antibodies to phosphoGSK3" (Tyr-279)/phospho-GSK3\$(Tyr-216) (the only antibody we found to be satisfactory) showed GSK3 staining predominantly in the nuclei, with much weaker staining in the cytoplasm (the site of glycogen metabolism) (Fig. 5A). Thus, GSK3 could to influence gene expression through phosphorylation of nuclear transcription factors. A nuclear pool of GSK3 has been detected in other cells [54], and nuclear GSK3 is reportedly increased by isoprenaline in adult cardiac myocytes [48]. However, we could not see any effect of insulin or ET-1 on the subcellular localisation of GSK3 in neonatal cells (data not shown).

Given the presence of GSK3 in cardiac myocyte nuclei (Fig. 5A), and that activation of PKB/Akt with consequent inhibition of GSK3 may represent a signalling pathway that promotes the changes in gene expression associated with cardiac myocyte hypertrophy [20,21], we examined the potential changes in AP1 transcription factor activity induced by 
inhibition of GSK3. GSK3 phosphorylates Jun family transcription factors at residues immediately N-terminal to their DNA binding domains and these phosphorylations negatively regulate their transactivating potentials [52]. For c-Jun, the decrease in transactivating ability may result from either decreasing DNA binding [72] or by promoting its degradation [73]. EMSAs with cardiac myocyte nuclear extracts showed that ET-1 increased binding of AP1 components to a DNA consensus sequence (Fig. 6A and B). This resulted from an increase in several AP1 components (Fig. 6). Although insulin or the GSK3 inhibitor 1-azakenpaullone also increased AP1 binding, this was less than that induced by ET-1 and derived principally from an increase in JunB binding. The differences between 1azakenpaullone and ET-1 on gene expression were further highlighted in our microarray experiments. It is clear from Table 1 that, although 1-azakenpaullone does cause changes in transcript abundances (three of which were confirmed by qPCR, see Fig. 7), these differ from those induced by ET-1. Thus, from the perspective of gene expression, cardiac myocyte hypertrophy induced by ET-1 is unlikely to be mediated by GSK3 to any significant extent.

Key aspects of the hypertrophic response are increases in cell size and myofibrillar content. Exposure of cardiac myocytes to 1-azakenpaullone for $24 \mathrm{~h}$ resulted in increases in cell area that resulted primarily from elongation rather than any increase in cell width (Fig. 5C - G). In contrast, cells exposed to established hypertrophic agonists such as phenylephrine (Fig. 5C - G) or ET-1 [62] displayed more uniform increases in both length and width. $\mathrm{Li}^{+}$ions have been used extensively as inhibitors of GSK3 (see, for example, [18]) and may be reasonably effective under physiological ATP/ $\mathrm{Mg}^{2+}$ concentrations [64]. Myocytes exposed to $\mathrm{Li}^{+}$also showed evidence of increased cell area and elongation (Fig. 5C and D) but the effect was less than with 1-azakenpaullone (possibly reflecting the relative potencies of the inhibitors). Confusingly, cardiospecific overexpression of GSK3\$ in vivo 
also causes an increase in length:width ratio [53]. Myocyte elongation has been previously observed with the gp130-coupled cytokine receptor agonist, cardiotrophin-1 [74]. As pointed out by Wollert et al. [74], because of the circumferential nature of myocyte orientation in the ventricular wall, such lengthening would be expected to be associated in vivo with a 'volumeoverload' hypertrophy (increased ventricular diameter with a proportional increase in ventricular wall thickness) or a dilated cardiomyopathy (ventricular dilatation with ventricular wall thinning). The pattern of hypertrophy induced by phenylephrine as predicted from Fig. 5C would be a 'pressure-overload' hypertrophy (ventricular wall thickening in the absence of changes in ventricular diameter). Although the kenpaullone derivatives are relatively specific for GSK3, other protein kinases that are potentially pro-hypertrophic (e.g. Rho kinases [75-77] and PDK1 [78,79]) are also inhibited [36,80]. However, it is the activation of these kinases that is considered to be pro-hypertrophic.

In conclusion, although ET-1 and insulin do both promote phosphorylation of GSK3 in cardiac myocytes (as previously reported [18]), the signalling pathways involved differ, with ET-1 operating primarily the ERK1/2 cascade and insulin signalling through PI3K. Inhibition of GSK3 by 1-azakenpaullone does produce changes in cardiac myocyte morphology, but these changes differ from those produced by ET-1 or phenylephrine. 1Azakenpaullone induces changes in gene expression but this are dissimilar from those induced by ET-1, which (in any case) promotes a much more extensive spectrum of changes (A. Clerk et al., manuscript submitted). Furthermore, although previous work in cardiac myocytes has focussed largely on GSK3\$, GSK3" is functional in these cells and should not be ignored. In the broader context, PKB/Akt is generally viewed as a 'pro-hypertrophic' kinase in the heart $[78,79]$. However, $\mathrm{PKB} / \mathrm{Akt}$ is strongly activated by insulin in cardiac myocytes [63], and GSK3" (Ser-21) and GSK3\$(Ser-9) become rapidly phosphorylated (Fig. 
1C-E), but insulin does not cause myocyte enlargement [62]. Viewed as a whole, these divergent results are difficult to rationalise in terms of the hypothesis that inhibition of GSK3 represents a primary stimulus to cardiac myocyte hypertrophy [20] or that ET-1-induced hypertrophy is mediated by inhibition of GSK3 [18].

\section{Acknowledgements}

This work was supported by British Heart Foundation Grant RG2001017 and by a Fondation Leducq 2004-2005 Transatlantic Network of Excellence Grant. We thank Laurence Game and Jayne L. Dennis of CSC Microarray Centre (Hammersmith Campus, Imperial College London) for assistance with the microarray experiments.

\section{References}

[1] N. Embi, D.B. Rylatt, P. Cohen, Eur. J. Biochem. 107 (1980) 519-527.

[2] P. Cohen, Nat. Rev. Mol. Cell Biol. 7 (2006) 867-873.

[3] A. Ali, K.P. Hoeflich, J.R. Woodgett, Chem. Rev. 101 (2001) 2527-2540.

[4] P. Cohen, S. Frame, Nat. Rev. Mol. Cell Biol. 2 (2001) 769-776.

[5] S. Frame, P. Cohen, Biochem. J. 359 (2001) 1-16.

[6] J.R. Woodgett, Sci. STKE 2001 (100) (2001) RE12.

[7] P. Cohen, M. Goedert, Nat. Rev. Drug Discov. 3 (2004) 479-487.

[8] C.Y. Logan, R. Nusse, Annu. Rev. Cell Dev. Biol. 20 (2004) 781-810.

[9] K.M. Cadigan, Y.I. Liu, J. Cell Sci. 119 (2006) 395-402.

[10] M.D. Gordon, R. Nusse, J. Biol. Chem. 281 (2006) 22429-22433. 
[11] K. Hughes, E. Nikolakaki, S.E. Plyte, N.F. Totty, J.R. Woodgett, EMBO J. 12 (1993) 803-808.

[12] Q.M. Wang, C.J. Fiol, A.A. DePaoli-Roach, P.J. Roach, J. Biol. Chem. 269 (1994) 14566-14574.

[13] A. Cole, S. Frame, P. Cohen, Biochem. J. 377 (2004) 249-255.

[14] T. Obata, M.B. Yaffe, G.G. Leparc, E.T. Piro, H. Maegawa, A. Kashiwagi, R. Kikkawa, L.C. Cantley, J. Biol. Chem. 275 (2000) 36108-36115.

[15] C. Sutherland, I.A. Leighton, P. Cohen, Biochem. J. 296 (1993) 15-19.

[16] L.M. Eisenberg, C.A. Eisenberg, ScentificWorldJournal 7 (2007) 161-176.

[17] C. Morisco, D. Zebrowski, G. Condorelli, P. Tsichlis, S.F. Vatner, J. Sadoshima, J. Biol. Chem. 275 (2000) 14466-14475.

[18] S. Haq, G. Choukroun, Z.B. Kang, H. Ranu, T. Matsui, A. Rosenzweig, J.D. Molkentin, A. Alessandrini, J. Woodgett, R. Hajjar, A. Michael, T. Force, J. Cell Biol. 151 (2000) 117-129.

[19] C.L. Antos, T.A. McKinsey, N. Frey, W. Kutschke, J. McAnally, J.M. Shelton, J.A. Richardson, J.A. Hill, E.N. Olson, Proc. Natl. Acad. Sci. U. S. A. 99 (2002) 907-912. [20] R. Kerkalä, K. Woulfe, T. Force, Trends Cardiovasc. Med. 17 (2007) 91-96. [21] P.H. Sugden, S.J. Fuller, S.C. Weiss, A. Clerk, Br. J. Pharmacol. in press (2007) [22] J. Sadoshima, S. Izumo, Annu. Rev. Physiol. 59 (1997) 551-571.

[23] L.H. Opie, P.J. Commerford, B.J. Gersh, M.A. Pfeffer, Lancet 367 (2006) 356-367. [24] P. Simpson, J. Clin. Invest. 72 (1983) 732-738.

[25] H.R. Lee, S.A. Henderson, R. Reynolds, P. Dunnmon, D. Yuan, K.R. Chien, J. Biol. Chem. 263 (1988) 7352-7358. 
[26] K. Iwaki, V.P. Sukhatme, H.E. Shubeita, K.R. Chien, J. Biol. Chem. 265 (1990) 13809-13817.

[27] H.E. Shubeita, P.M. McDonough, A.N. Harris, K.U. Knowlton, C.C. Glembotski, J.H.

Brown, K.R. Chien, J. Biol. Chem. 265 (1990) 20555-20562.

[28] C. Morisco, J. Sadoshima, B. Trimarco, R. Arora, D.E. Vatner, S.F. Vatner, Am. J.

Physiol. Heart Circ. Physiol. 284 (2003) H1043-H1047.

[29] P.H. Sugden, A. Clerk, J. Mol. Med. 76 (1998) 725-746.

[30] P.H. Sugden, Ann. Med. 33 (2001) 611-622.

[31] B.J. Wilkins, J.D. Molkentin, Biochem. Biophys. Res. Commun. 322 (2004) 1178-1191.

[32] T. Zhang, J.H. Brown, Cardiovasc. Res. 63 (2004) 476-486.

[33] J. Heineke, J.D. Molkentin, Nat. Rev. Mol. Cell Biol. 7 (2006) 589-600.

[34] T.A. McKinsey, Cardiovasc. Res. 73 (2007) 667-677.

[35] M. Frödin, S. Gammeltoft, Mol. Cell. Endocrinol. 151 (1999) 65-77.

[36] C. Kunick, K. Lauenroth, M. Leost, M. Meijer, T. Lemcke, Bioorg. Med. Chem. Lett. 14 (2004) 413-416.

[37] M.A. Bogoyevitch, A. Clerk, P.H. Sugden, Biochem. J. 309 (1995) 437-443.

[38] A. Clerk, P.H. Sugden, Biochem. J. 325 (1997) 801-810.

[39] M.M. Bradford, Anal. Biochem. 72 (1976) 248-254.

[40] A. Clerk, M.A. Bogoyevitch, M.B. Andersson, P.H. Sugden, J. Biol. Chem. 269 (1994) 32848-32857.

[41] D.A. Cross, D.R. Alessi, J.R. Vandenheede, H.E. McDowell, H.S. Hundal, P. Cohen, Biochem. J. 303 (1994) 21-26.

[42] T.J. Kemp, H.C. Causton, A. Clerk, Biochem. Biophys. Res. Commun. 307 (2003) 416-421. 
[43] M.F. Favata, K.Y. Horiuchi, E.J. Manos, A.J. Daulerio, D.A. Stradley, W.S. Feeser, D.E. Van Dyk, W.J. Pitts, R.A. Earl, F. Hobbs, R.A. Copeland, R.L. Magolda, P.A. Scherle, J.M. Trzaskos, J. Biol. Chem. 273 (1998) 18623-18632.

[44] C.J. Vlahos, W Matter,F,, K.Y. Hui, R.F. Brown, J. Biol. Chem. 269 (1994) 5241-5248.

[45] H. Murai, M. Okazaki, A. Kikuchi, FEBS Lett. 392 (1996) 153-160.

[46] M. Lesort, R.S. Jope, G.V.W. Johnson, J. Neurochem. 72 (1999) 576-584.

[47] R.V. Bhat, J. Shanley, M.P. Correll, W.E. Fieles, R.A. Keith, C.W. Scott, C.M. Lee, Proc. Natl. Acad. Sci. U. S. A. 97 (2000) 11074-11079.

[48] B. Menon, J.N. Johnson, R.S. Ross, M. Singh, K. Singh, J. Mol. Cell. Cardiol. 42 (2007) 653-661.

[49] M. Shaw, P. Cohen, D.R. Alessi, FEBS Lett. 416 (1997) 307-311.

[50] E. Shaulian, M. Karin, Oncogene 20 (2001) 2390-2400.

[51] E. Shaulian, M. Karin, Nat. Cell Biol. 4 (2002) E131-E136.

[52] E. Nikolakaki, P.J. Coffer, R. Hemelsoet, J.R. Woodgett, L.H. Defize, Oncogene 8 (1993) 833-840.

[53] A. Michael, S. Haq, X. Chen, E. Hsich, L. Cui, B. Walters, Z. Shao, K. Bhattacharya, H. Kilter, G. Huggins, M. Andreucci, M. Periasamy, R.N.. Solomon, R. Liao, R. Patten, J. D. Molkentin, T. Force, J. Biol. Chem. 279 (2004) 21383-21393.

[54] R.S. Jope, G.V.W. Johnson, Trends Biochem. Sci. 29 (2004) 95-102.

[55] E.J. McManus, K. Sakamoto, L.J. Armit, L. Ronaldson, N. Shpiro, R. Marquez, D.R. Alessi, EMBO J. 24 (2005) 1571-1583.

[56] A. Mora, K. Sakamoto, E.J. McManus, D.R. Alessi, FEBS Lett. 579 (2005) 3632-3638. [57] M.H. Linag, D.M. Chuang, J. Biol. Chem. 281 (2006) 30479-30484.

[58] M.H. Linag, D.M. Chuang, J. Biol. Chem. 282 (2007) 3904-3917. 
[59] S.P. Shevtsov, S. Haq, T. Force, Cell Cycle 5 (2006) 2295-2300.

[60] M.A. Bogoyevitch, P.E. Glennon, M.B. Andersson, A. Clerk, A. Lazou, C.J. Marshall, P.J. Parker, P.H. Sugden, J. Biol. Chem. 269 (1994) 1110-1119.

[61] F.H. Pham, S.M. Cole, A. Clerk, Adv. Enzyme Regul. 41 (2001) 73-86.

[62] A. Clerk, I.-K.S. Aggeli, K. Stathopoulou, P.H. Sugden, Cell. Signal. 18 (2006) 225-235.

[63] F.H. Pham, P.H. Sugden, A. Clerk, Circ. Res. 86 (2000) 1252-1258.

[64] S.P. Davies, H. Reddy, M. Caivano, P. Cohen, Biochem. J. 351 (2000) 95-105.

[65] S.I. Gharbi, M.J. Zvelebil, S.J. Shuttleworth, T. Hancox, N. Saghir, J.F. Timms, M.D. Waterfield, Biochem. J. 404 (2007) 15-21.

[66] S. Haq, A. Michael, M. Andreucci, K. Bhattacharya, P. Dotto, B. Walters, J. Woodgett, H. Kilter, T. Force, Proc. Natl. Acad. Sci. U. S. A. 100 (2003) 4610-4615.

[67] X. Chen, S.P. Shevtsov, E. Hsich, L. Cui, S. Haq, M. Aronovitz, R. Kerkalä, J.D. Molkentin, R. Liao, R.N. Salomon, R. Patten, T. Force, Mol. Cell. Biol. 26 (2006) 4462-4473.

[68] C. Morisco, K. Seta, S.E. Hardt, Y. Lee, S.F. Vatner, J. Sadoshima, J. Biol. Chem. 276 (2001) 28586-28597.

[69] C. Badorff, F.H. Seeger, A.M. Zeiher, S. Dimmeler, Circ. Res. 97 (2005) 645-654.

[70] S.E. Hardt, H. Tomita, H.A. Katus, J. Sadoshima, Circ. Res. 94 (2004) 926-935.

[71] S. Frame, P. Cohen, R.M. Biondi, Mol. Cell 7 (2001) 1321-1327.

[72] W.J. Boyle, T. Smeal, L.H.K. Defize, P. Angel, J.R. Woodgett, M. Karin, T. Hunter, Cell 64 (1991) 573-584.

[73] W. Wei, J. Jin, S. Schlisio, J.W. Harper, W.G. Kaelin, Jr., Cancer Cell 8 (2005) 25-33. 
[74] K.C. Wollert, T. Taga, M. Saito, M. Narazaki, T. Kishimoto, C.C. Glembotski, A.B.

Vernallis, J.K. Heath, D. Pennica, W.I. Wood, K.R. Chien, J. Biol. Chem. 271 (1996) 9535-9545.

[75] M. Hoshijima, V.P. Sah, Y. Wang, K.R. Chien, J.H. Brown, J. Biol. Chem. 273 (1998) 7725-7730.

[76] K. Kuwahara, Y. Saito, O. Nakagawa, I. Kishimoto, M. Harada, E. Ogawa, Y.

Miyamoto, I. Hamanaka, N. Kajiyama, N. Takahashi, T. Izumi, R. Kawakami, N. Tamura, Y. Ogawa, K. Nakao, FEBS Lett. 452 (1999) 314-318.

[77] J.H. Brown, D.P. Del Re, M.A. Sussman, Circ. Res. 98 (2006) 730-742.

[78] T. Matsui, A. Rosenzweig, J. Mol. Cell. Cardiol. 38 (2005) 63-71.

[79] I. Shiojima, K. Walsh, Genes Dev. 20 (2006) 3347-3365.

[80] J. Bain, H. McLauchlan, M. Elliott, P. Cohen, Biochem. J. 371 (2003) 199-204.

\section{Figure Legends}

Fig. 1. Time courses of phosphorylation of GSK3" (Ser-21) and GSK3\$(Ser-9) in cardiac myocytes. Myocytes were exposed to $100 \mathrm{nM}$ ET-1, 1 : M PMA, or $300 \mathrm{nM}$ insulin for the times indicated (A-E), or to ET-1, PMA or insulin for 20 min (F and G). Phosphorylation of GSK3" (Ser-21) or GSK3\$(Ser-9) was estimated by immunoblotting (20 : g protein per lane) (A - C and F, upper panels). To detect total GSK3" and GSK3\$ (used as loading controls), antibodies were mixed (A - C and F, lower panels). D, E and G, Phosphorylation of GSK3" (Ser-21) or GSK3\$(Ser-9) was quantified by calibrated scanning densitometry for 3 (F) or 4 (D and E) separate preparations of myocytes. Results are means \pm S.E.M. ${ }^{*} \mathrm{p}<0.01$ 
relative to control; $\# \mathrm{p}<0.05$ relative to insulin (one-way ANOVA with Tukey post-test, $\mathrm{n}=3$ independent myocyte preparations).

Fig. 2. Effects of inhibition of PI3K or the ERK1/2 cascade on the phosphorylation of GSK3" (Ser-21) or GSK3\$(Ser-9) by 100 nM ET-1, 1 : M PMA, or 300 nM insulin in cardiac myocytes. Myocytes were pre-exposed to 50 : M LY294002 or to 10 : M U0126 for 10 min before agonists were added for a further 10 min. Phosphorylation of GSK3" (Ser-21) or GSK3\$(Ser-9) was estimated by immunoblotting (20: g protein per lane) (A - C, upper panels). To detect total GSK3" and GSK3\$ (used as loading controls), antibodies were mixed (A - C, lower panels). Phosphorylation of GSK3" (Ser-21) (D) or GSK3\$(Ser-9) (E) was quantified by calibrated scanning densitometry. Results are means \pm S.E.M. ${ }^{*} \mathrm{p}<0.05$ relative to control; \# $\mathrm{p}<0.05$ relative to agonist alone [repeated measures one-way ANOVA with Tukey post-test; $\mathrm{n}=4$ (ET-1 or insulin) or n=5 (PMA) independent myocyte preparations].

Fig. 3. Time courses of phosphorylation of GSK3" (Tyr-279) and GSK3\$(Tyr-216) in cardiac myocytes. Myocytes were exposed to $100 \mathrm{nM}$ ET-1 (A), 1 : M PMA (B), $300 \mathrm{nM}$ insulin (C) or 10 : M 1-azakenpaullone (D and E) for the times indicated. Phosphorylation of GSK3" (Tyr-279) and GSK3\$(Tyr-216) was estimated by immunoblotting (20 : g protein per lane) (A - D, upper panels). To detect total GSK3" and GSK3\$ (used as loading controls), antibodies were mixed (A - D, lower panels). The experiments were repeated 3 times with similar results. (E) Phosphorylation of GSK3" (Tyr-279) and GSK3\$(Tyr-216) following exposure of cardiac myocytes to 1-azakenpaullone was quantified by calibrated scanning 
densitometry. Results are means \pm S.E.M. ${ }^{*} \mathrm{p}<0.01$, \# $\mathrm{p}<0.05$ relative to control (one-way ANOVA with Dunnett post-test for $n=3$ independent myocyte preparations).

Fig. 4. MonoS FPLC separation of GSK3.. Myocytes (4 dishes per column) were unstimulated (Control), or exposed to $100 \mathrm{nM}$ ET-1 or $300 \mathrm{nM}$ insulin for $10 \mathrm{~min}$. (A) Elution of GSK3 activity from MonoS columns. The linear $\mathrm{NaCl}$ gradient is shown as a dashed line. (B) Elution profiles of GSK3 activity (from panel A) were integrated. Activities are expressed relative to control values and are means \pm S.E.M. ${ }^{*} \mathrm{p}<0.01$ relative to control, \# $\mathrm{p}<0.05$ relative to insulin (one-way ANOVA with Dunnett post-test for $\mathrm{n}=3$ independent myocyte preparations). (C-E) Fractions (20: l) from control myocytes, or from myocytes exposed to ET-1 or insulin were immunoblotted for phospho-GSK3" (Tyr-279)/phosphoGSK3\$(Tyr-216) (C), GSK3\$ (D), or phospho-GSK3" (Ser-21)/phospho-GSK3\$(Ser-9) (E). All fractions were surveyed initially, but only fractions containing GSK3 are shown. The experiment was repeated 3 times with identical results. (F) Direct comparison of phosphoGSK3" (Ser-21)/phospho-GSK3\$(Ser-9) (upper panels) or GSK3\$ (lower panels) in fractions from control cells, or from cells exposed to ET-1 or insulin. The experiment was repeated 3 times with identical results.

Fig. 5. Subcellular localisation of GSK3 in cardiac myocytes and effects of GSK3 inhibition on cardiac myocyte morphology. GSK3 was detected by immunostaining with anti-phosphoGSK3" (Tyr-279)/phospho-GSK3\$(Tyr-216) (A), and nuclei were counterstained with Hoechst 33258 (B). (C) Control myocytes (top left), or myocytes exposed to 100 : M phenylephrine (top right), $10 \mathrm{mM} \mathrm{LiCl} \mathrm{(bottom} \mathrm{right),} \mathrm{or} 10$ : M 1-azakenpaullone (bottom left) for $24 \mathrm{~h}$ were stained for cardiac troponin T. Images are representative areas from 4 
independent experiments from separate myocyte preparations. Areas (D), maximum lengths (E), maximum widths (F), and maximum length:maximum width ratios (G) of control cells, and cells exposed to phenylephrine, 1-azakenpaullone or (for cell areas) $\mathrm{LiCl}$ were measured for at least 100 myocytes in each experiment. Results are means \pm S.E.M. for $n=4$ (D, areas) or $n=3$ ( $E$ - G, maximum lengths/widths/ratios) independent myocyte preparations for which each value was taken as the mean of all the cells measured in a particular experiment. * $\mathrm{p}<0.05$ or $* * \mathrm{p}<0.01$ relative to controls, $\# \mathrm{p}<0.01$ relative to 1-azakenpaullone (repeated measures one-way ANOVA with Tukey post-test, $n=4$ (D) or $n=3$ (E and G) independent myocyte preparations).

Fig. 6. Electrophoretic mobility shift assays. (A) Binding of nuclear extract proteins from cardiac myocytes exposed to 10 : M 1-azakenpaullone, $300 \mathrm{nM}$ insulin, or $100 \mathrm{nM}$ ET-1 for 30 or 60 min to an AP1 oligonucleotide probe. (B) AP1 binding (panel A) was quantified by calibrated scanning densitometry. Results are means \pm S.E.M. ${ }^{*} \mathrm{p}<0.05$ relative to control (one-way ANOVA with Dunnett post-test) for $n=3$ separate preparations of myocytes. (C) Supershift EMSA with the indicated antibodies was used to identify proteins bound to the AP1 probe. Myocyes were exposed to 1-azakenpaullone, ET-1 or insulin for 60 min. (D and E) Effects of $10:$ M 1-azakenpaullone (D) or $300 \mathrm{nM}$ insulin (E) on JunB abundance as assessed by immunoblotting of nuclear extracts (20: g protein per lane) (upper panels). Bands were quantified by calibrated scanning densitometry (lower panels). Results are means \pm S.E.M. for $n=3$ separate preparations of myocytes.

Fig. 7. qPCR analysis of the effects of 1-azakenpaullone on mRNA expression of prostaglandin-endoperoxide synthase 2 (Ptgs2) (A), cyclin G2 (B), or adrenomedullin (C). 
Cardiac myocytes were exposed to 10 : M 1-azakenpaullone for the times indicated. Total RNA was extracted and mRNA expression measured by qPCR. Results (normalised to Gapd) are means \pm S.E.M. for $n=3$ (cyclin G2) or $n=4$ (Ptgs2 or adrenomedullin) independent myocyte preparations. 
Table 1. Effects of 1-azakenpaullone on gene expression in cardiac myocytes. Cardiac myocytes were unstimulated, or exposed to $10 \mu \mathrm{M}$ 1-azakenpaullone (1-AKP) or $100 \mathrm{nM}$ ET-1 for $1 \mathrm{~h}$. RNA was extracted and gene expression profiling performed with Affymetrix rat genome 2302.0 arrays with downstream analysis using GeneSpring GX 7.3.1. Genes with a (p) post-script in section (d) are predicted. Genes were identified with significant differences in expression induced by 1azakenpaullone relative to unstimulated controls (FDR $<0.05$; 2-fold minimum difference). Results are means \pm S.E.M. for the expression relative to unstimulated controls ( $n=3$ independent sets of samples, each prepared from at least 3 separate myocyte preparations).

\begin{tabular}{|c|c|c|c|c|}
\hline Gene & Gene Title (probe) & Probe & 1-AKP & ET-1 \\
\hline \multicolumn{5}{|c|}{ (a) 1-Azakenpaullone endothelin } \\
\hline Ddit4 & DNA-damage-inducible transcript 4 & 1368025_at & $0.35 \pm 0.06$ & $0.16 \pm 0.01$ \\
\hline Ppp1r3b & Protein phosphatase 1 , regulatory subunit 3B & 1384262_at & $0.46 \pm 0.04$ & $0.33 \pm 0.04$ \\
\hline \multicolumn{5}{|c|}{ (b) Endothelin > 1-Azakenpaullone } \\
\hline & FBJ murine osteosarcoma viral oncogene & & & \\
\hline Fos & homolog & 1375043_at & $2.00 \pm 0.15$ & $12.93 \pm 1.61$ \\
\hline Nr4a1 & Nuclear receptor subfamily 4 , group A, member 1 & $1386935^{-}$at & $2.30 \pm 0.46$ & $19.11 \pm 0.91$ \\
\hline Lif & Leukemia inhibitory factor & 1393728 at & $2.57 \pm 0.56$ & $10.93 \pm 1.21$ \\
\hline Ptgs2 & Prostaglandin-endoperoxide synthase 2 & 1368527_at & $3.37 \pm 0.95$ & $14.85 \pm 1.84$ \\
\hline FosB & FBJ osteosarcoma oncogene B & 1373759_at & $4.68 \pm 1.50$ & $117.1 \pm 23.1$ \\
\hline \multicolumn{5}{|c|}{ (c) 1-Azakenpaullone opposite to endothelin } \\
\hline Errfi1 & ERBB receptor feedback inhibitor 1 & 1373093_at & $0.32 \pm 0.02$ & $5.41 \pm 0.48$ \\
\hline Dusp1 & Dual specificity phosphatase 1 & 1368146_at & $0.38 \pm 0.04$ & $2.33 \pm 0.10$ \\
\hline KIf6 & Kruppel-like factor 6 & 1387060_at & $0.39 \pm 0.05$ & $4.41 \pm 0.56$ \\
\hline Gadd45b & $\begin{array}{l}\text { Growth arrest and DNA-damage-inducible } 45 \text { beta } \\
\text { Cbp/p300-interacting transactivator, with Glu/Asp- }\end{array}$ & 1372016_at & $0.44 \pm 0.05$ & $1.69 \pm 0.13$ \\
\hline Cited2 & rich carboxy-terminal domain, 2 & 1367602_at & $0.46 \pm 0.03$ & $4.69 \pm 0.17$ \\
\hline \multicolumn{5}{|c|}{ (d) Endothelin not significantly changed } \\
\hline Adm & Adrenomedullin & 1387219_at & $0.27 \pm 0.01$ & $0.88 \pm 0.09$ \\
\hline Ccng2 (p) & Cyclin G2 & 1371953_at & $0.32 \pm 0.05$ & $0.60 \pm 0.05$ \\
\hline Pnrc1 & Proline rich 2 & 1370381_at & $0.39 \pm 0.07$ & $1.01 \pm 0.07$ \\
\hline Pfkfb3 & 6-Phosphofructo-2-kinase & 1390391_at & $0.40 \pm 0.06$ & $0.70 \pm 0.10$ \\
\hline Ptger4 & Prostaglandin E receptor 4 & 1393638_at & $0.43 \pm 0.05$ & $1.32 \pm 0.24$ \\
\hline Rgs4 & $\begin{array}{l}\text { Regulator of G-protein signaling } 4 \\
\text { Gonadotropin inducible ovarian transcription }\end{array}$ & 1368505_at & $0.43 \pm 0.06$ & $0.81 \pm 0.09$ \\
\hline Giot1 & factor 1 & 1368775_at & $0.45 \pm 0.08$ & $0.66 \pm 0.19$ \\
\hline Sox18 & SRY-box containing gene 18 & 1381971_at & $0.48 \pm 0.01$ & $0.92 \pm 0.07$ \\
\hline Exo1 (p) & Exonuclease 1 & 1385733_at & $0.48 \pm 0.05$ & $0.86 \pm 0.13$ \\
\hline Rkhd3 (p) & Ring finger and $\mathrm{KH}$ domain containing 3 & 1380682_at & $0.48 \pm 0.03$ & $1.02 \pm 0.12$ \\
\hline Spry1 (p) & Sprouty homolog 1 (Drosophila) & 1379380_at & $0.49 \pm 0.05$ & $1.00 \pm 0.08$ \\
\hline Fzd8 & Frizzled homolog 8 (Drosophila) & 1383721_at & $0.49 \pm 0.05$ & $1.00 \pm 0.04$ \\
\hline Tnf & Tumor necrosis factor & 1391384_at & $0.50 \pm 0.08$ & $0.92 \pm 0.10$ \\
\hline Isl2 & Insulin related protein 2 & 1369530_at & $2.04 \pm 0.27$ & $1.64 \pm 0.40$ \\
\hline
\end{tabular}


Markou et al. Figure 1.

A B C

$\begin{array}{llllllllllllllllllll}\text { Time }(\mathrm{min}) & 0 & 2 & 5 & 10 & 20 & 30 & 0 & 2 & 5 & 10 & 20 & 30 & 0 & 1 & 2 & 5 & 10 & 20 & 30\end{array}$

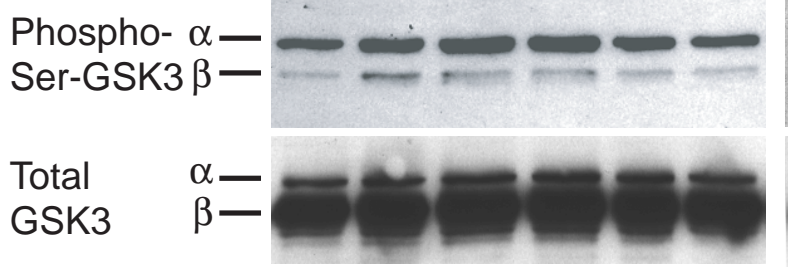

ET-1

$\mathrm{D}$

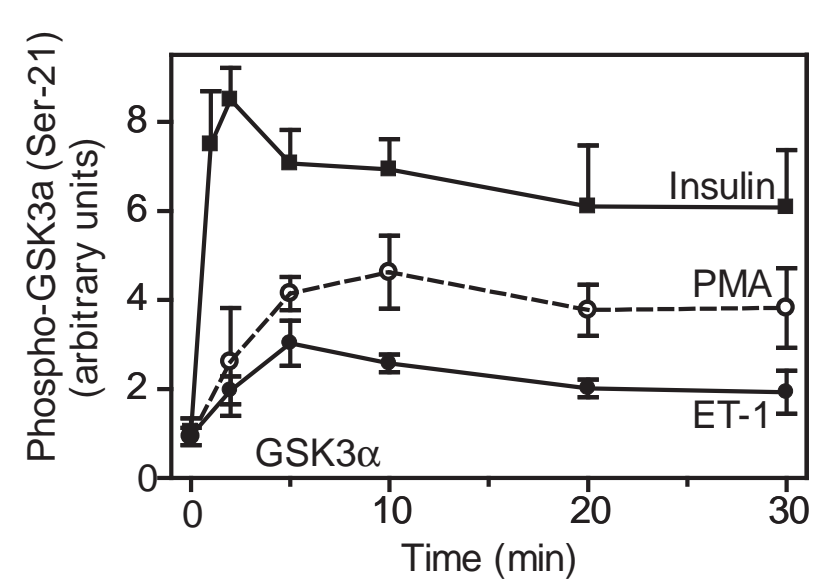

F

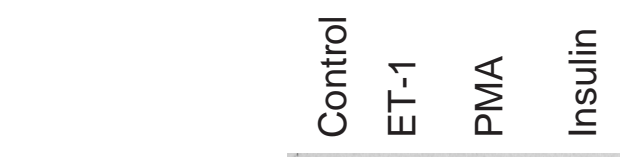

Phospho- $\alpha$ -

Ser-GSK3 $\beta$ -

Total

GSK3
PMA

Insulin

$\mathrm{E}$

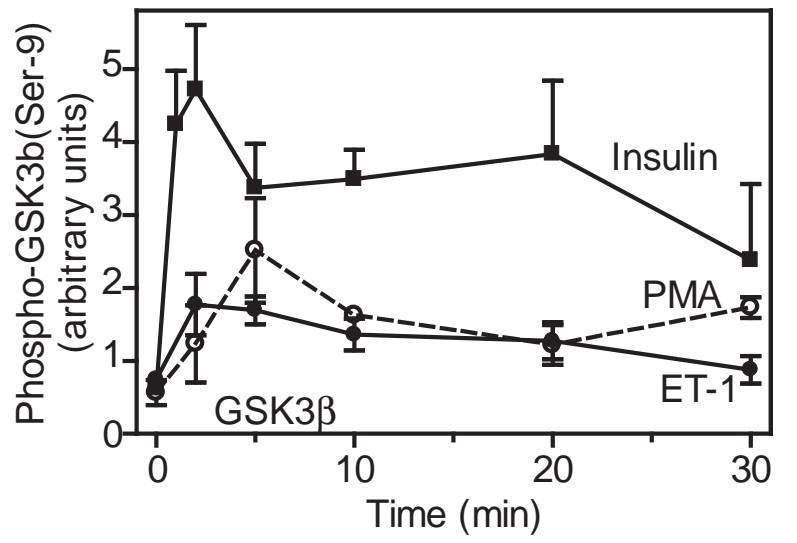

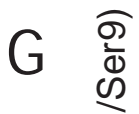

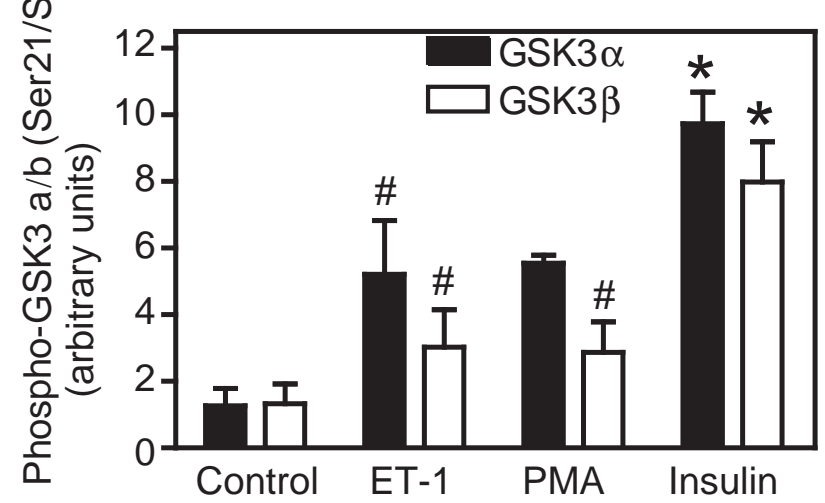


Markou et al. Figure 2.
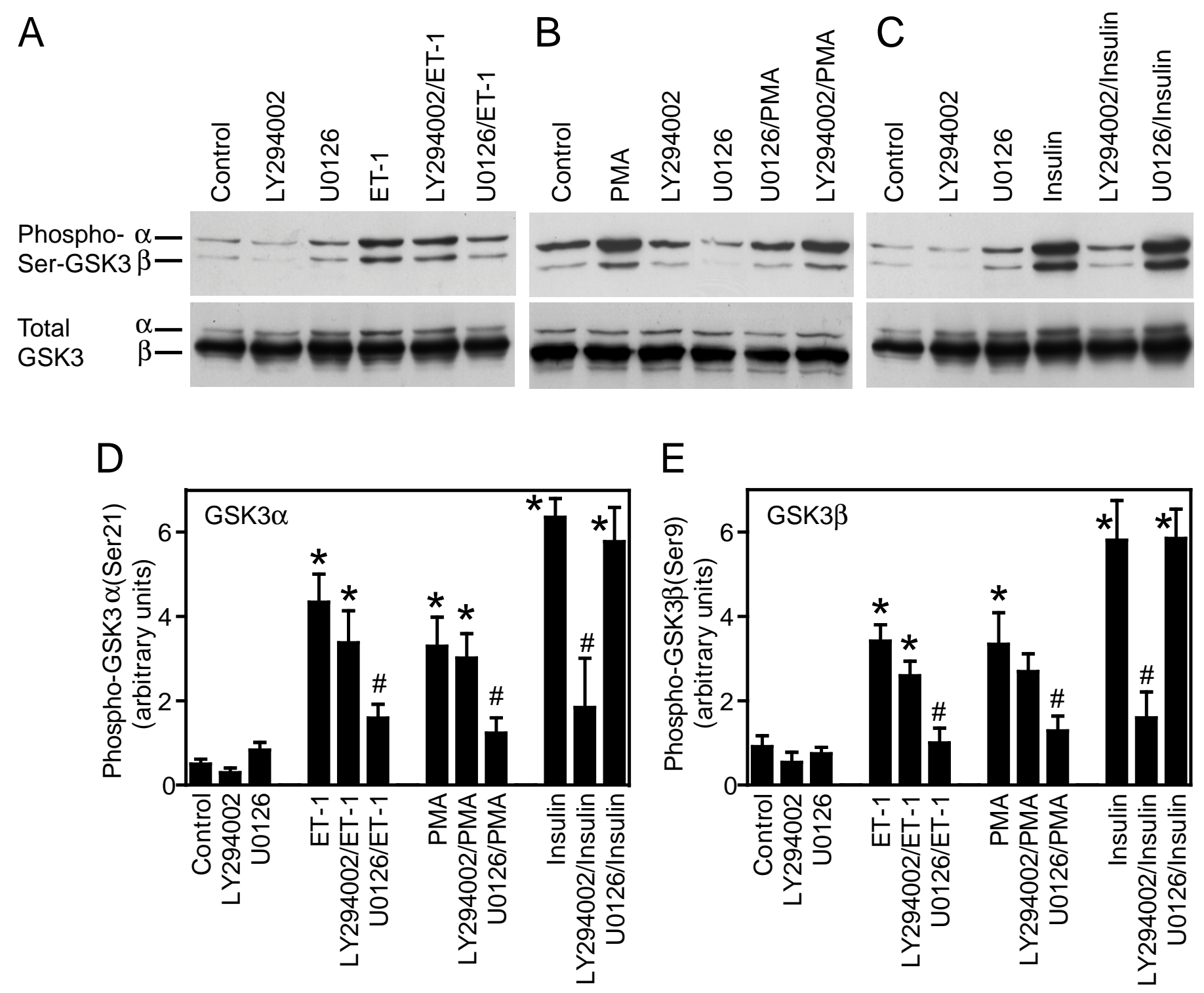
Markou et al. Figure 3.

A

B

C

$\begin{array}{llllllllllllllllllll}\text { Time }(\min ) & 0 & 2 & 5 & 10 & 20 & 30 & 0 & 2 & 5 & 10 & 20 & 30 & 0 & 1 & 2 & 5 & 10 & 20 & 30\end{array}$

Phospho- $\alpha$ -

Tyr-GSK3 $\beta-$

$\begin{array}{ll}\text { Total } & \alpha= \\ \text { GSK3 } & \beta-\end{array}$

ET-1

PMA

Insulin

D

Time (h) $\quad 0 \quad 2 \quad 2 \quad 4 \quad 6 \quad 8 \quad 12$

Phospho- $\alpha$ -

Tyr-GSK3 $\beta$ -

Total

GSK3 $\beta-$

E

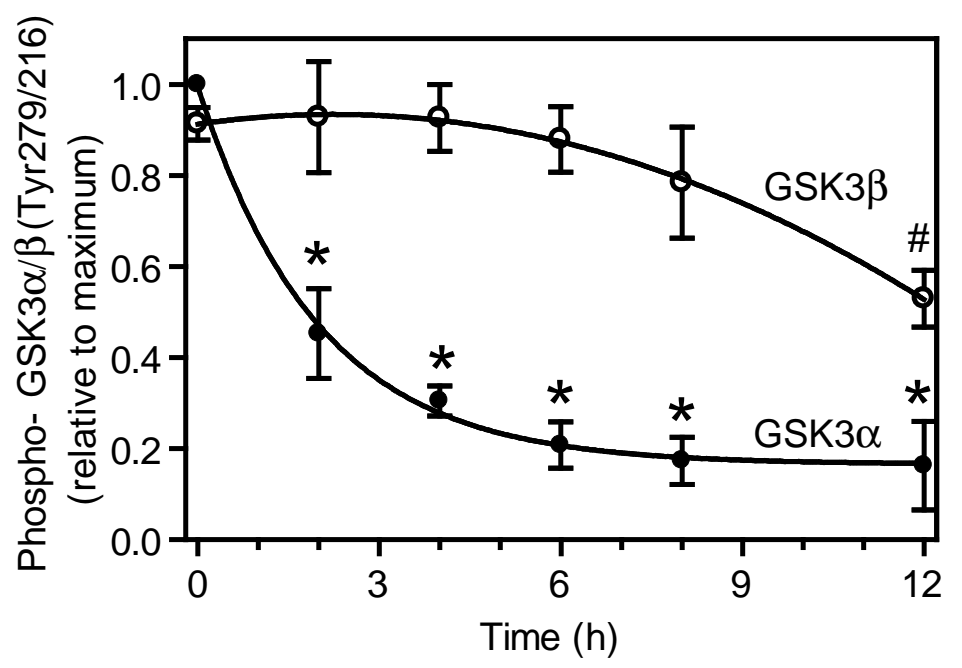


A

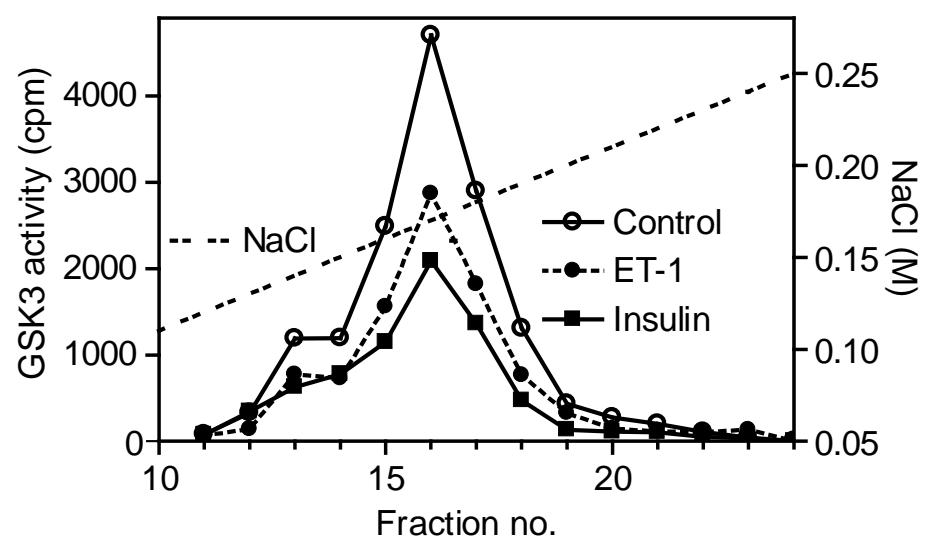

$\mathrm{B}$

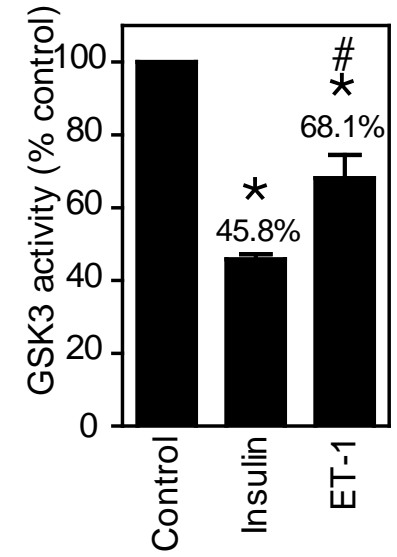

C

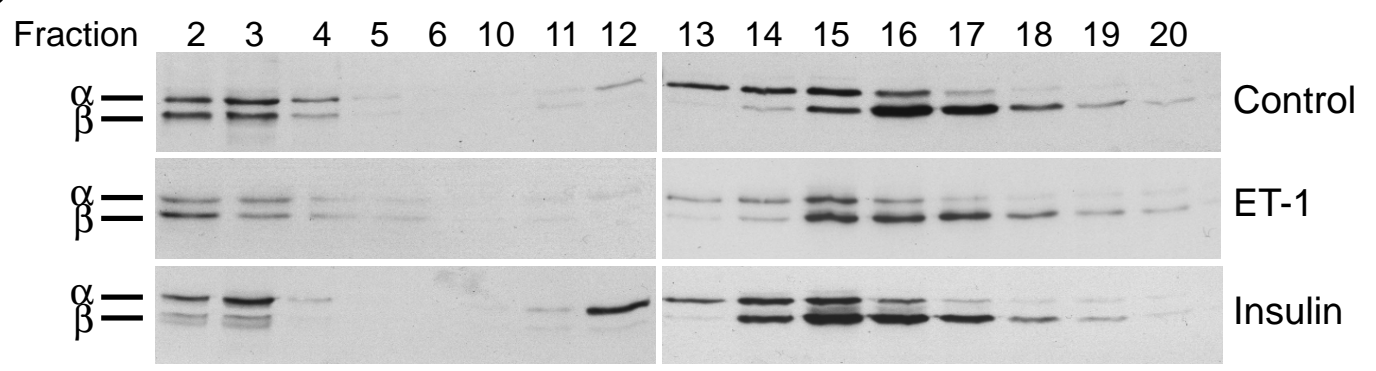

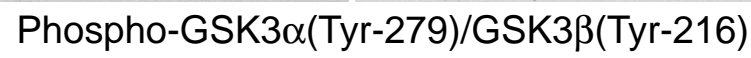

D

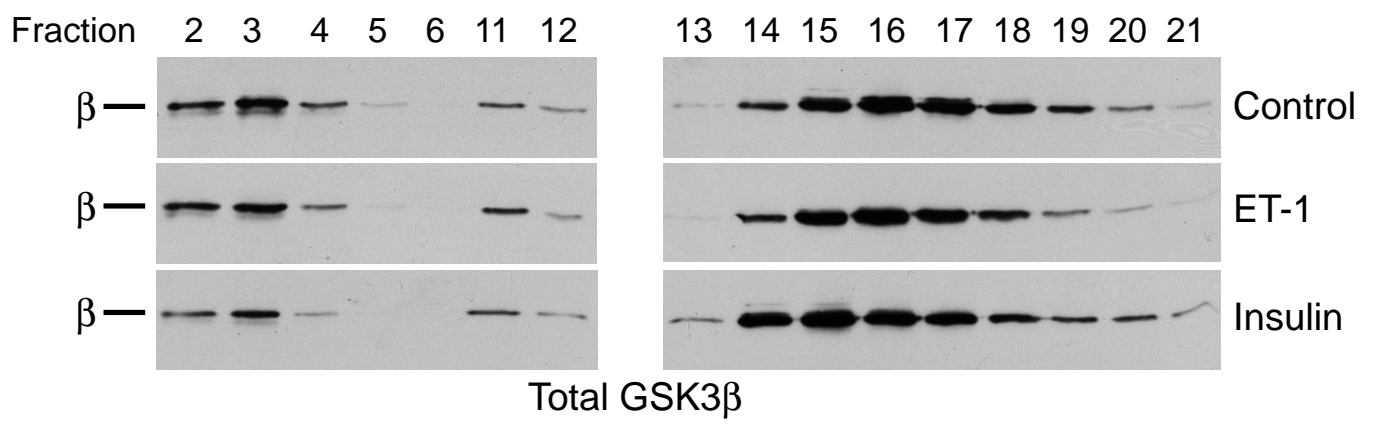

$E$

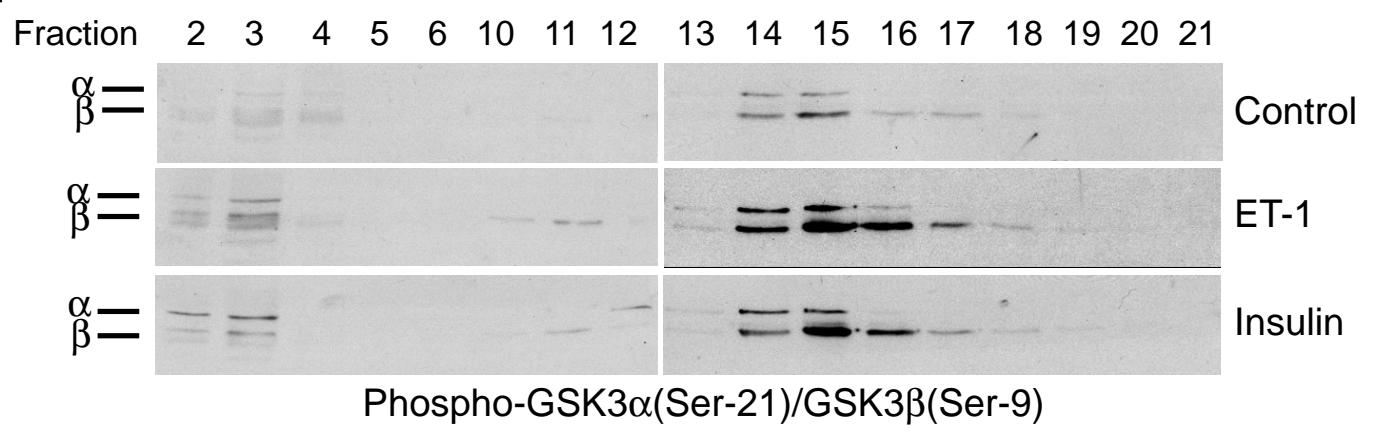

F

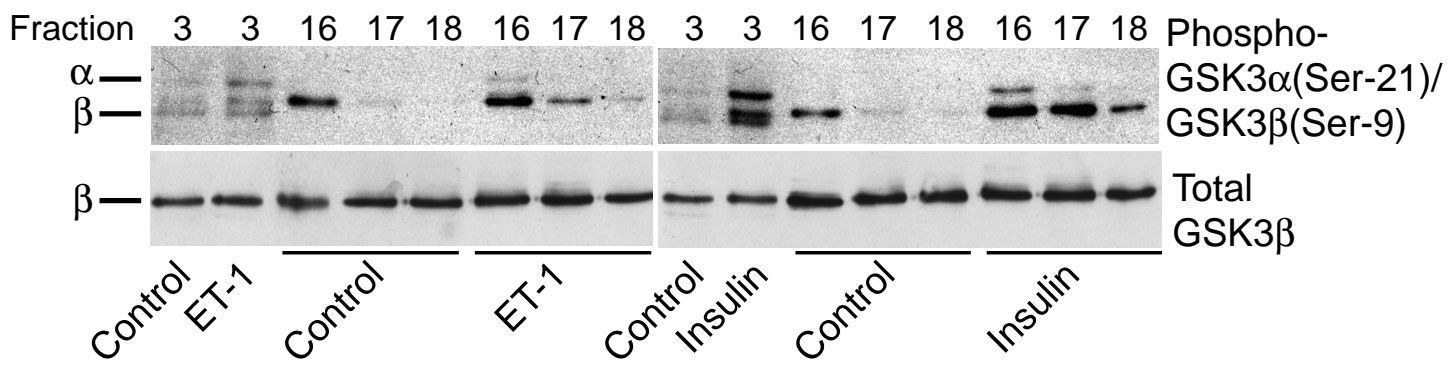


Markou et al. Figure 5.

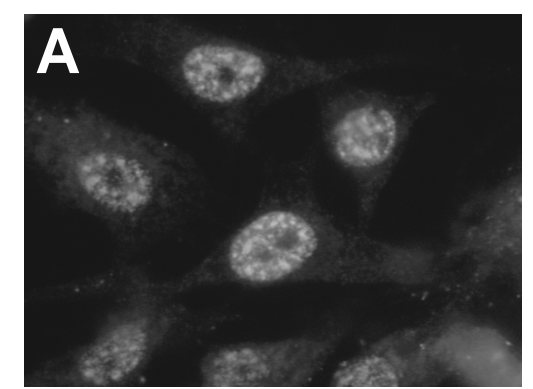

GSK3 $\alpha / \beta(T y r-279 / 216)$

C
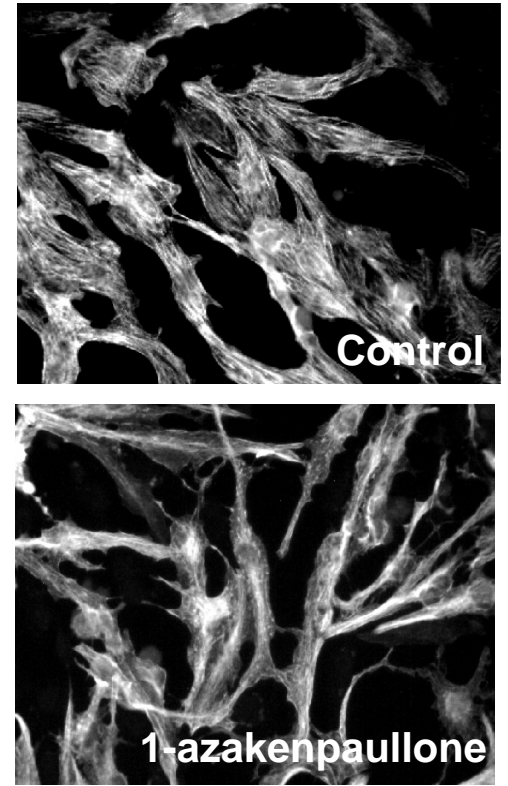

D

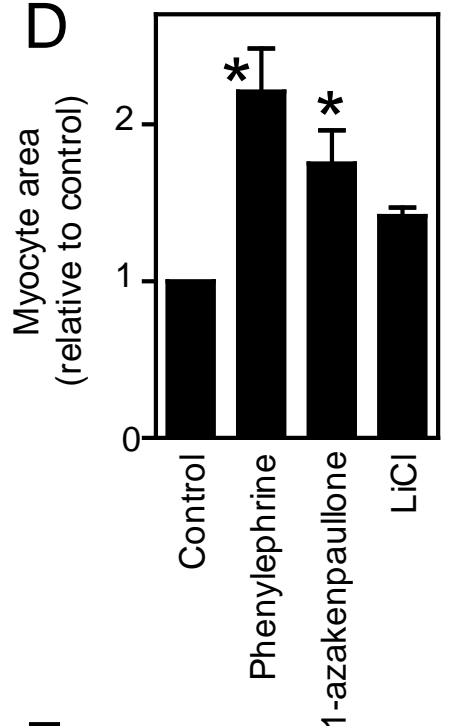

F

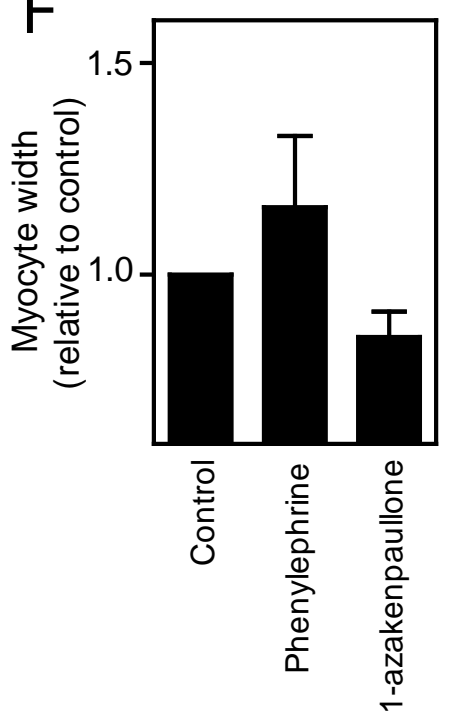

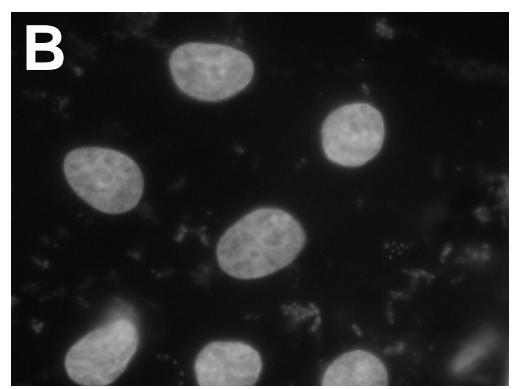

Hoescht 33258
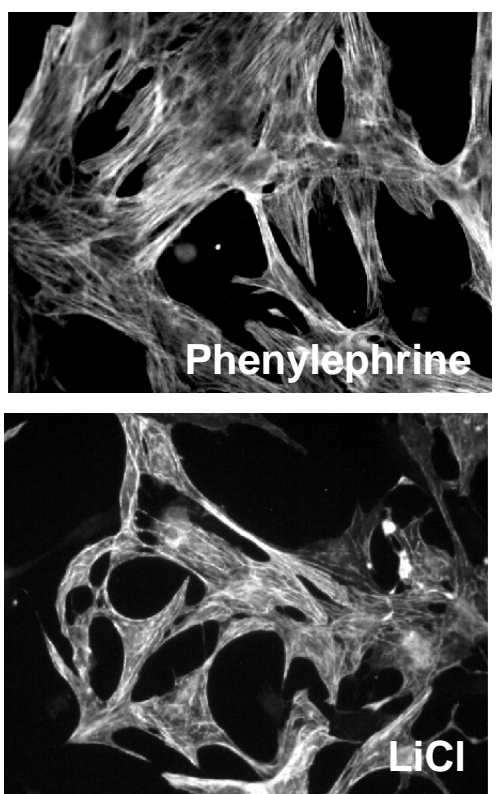

E

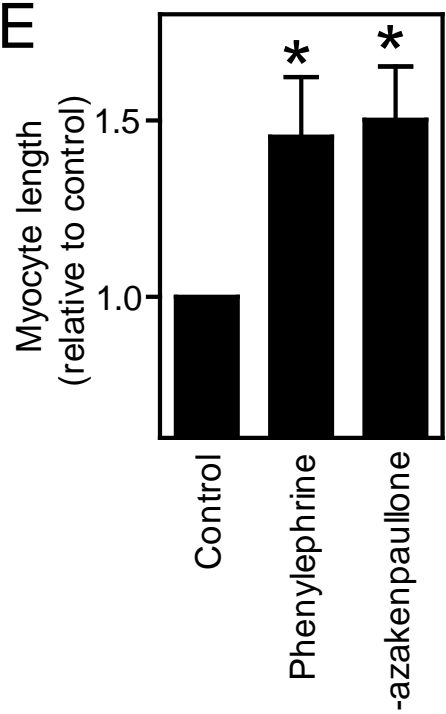

G

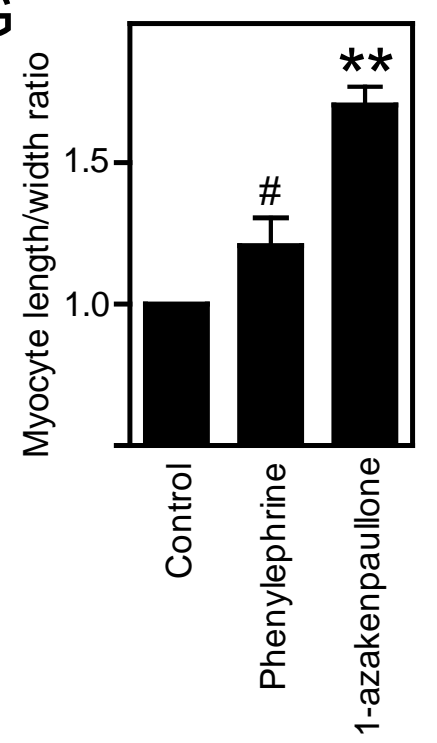


Markou et al. Figure 6

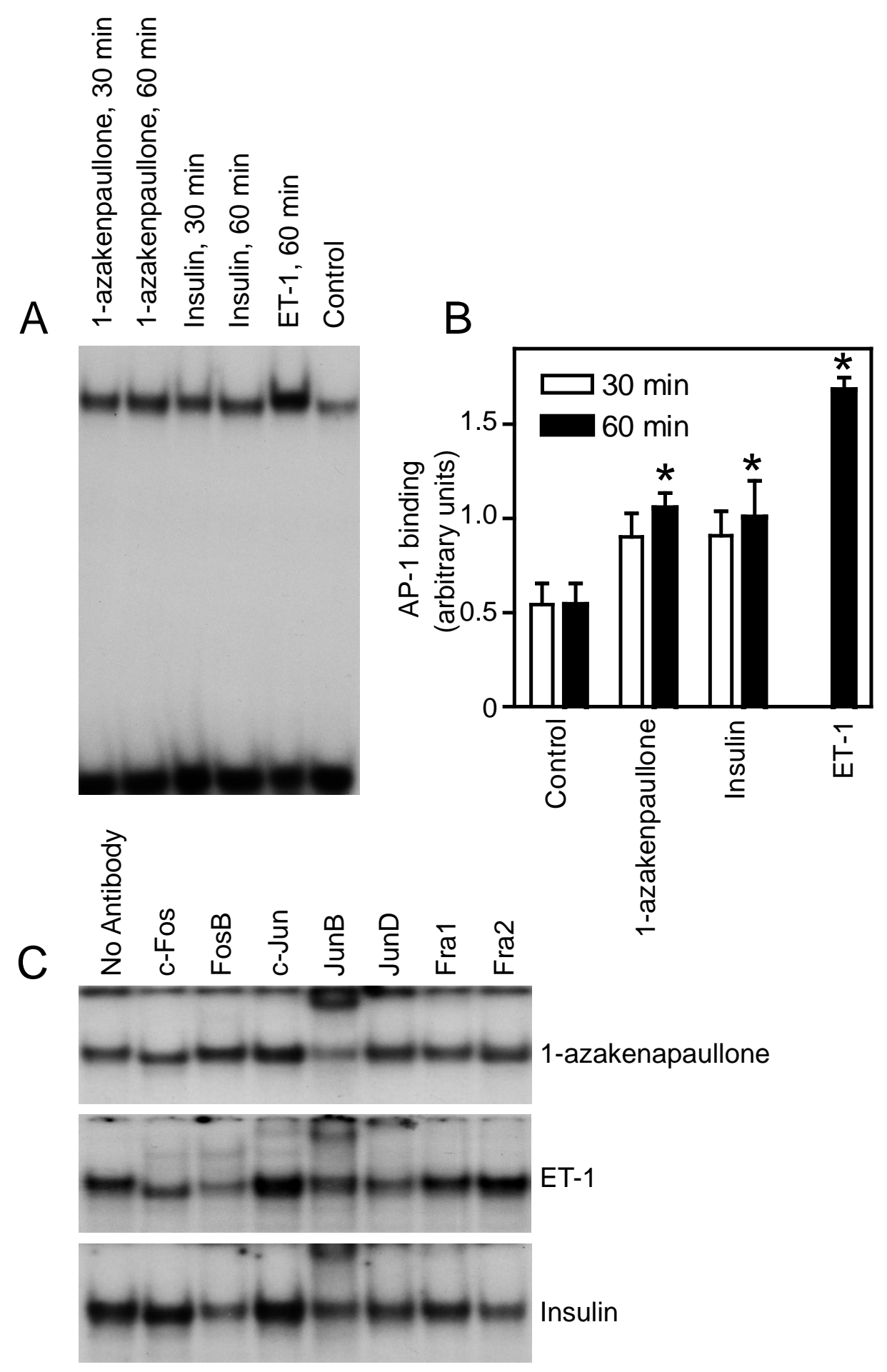


Markou et al. Figure 7

A

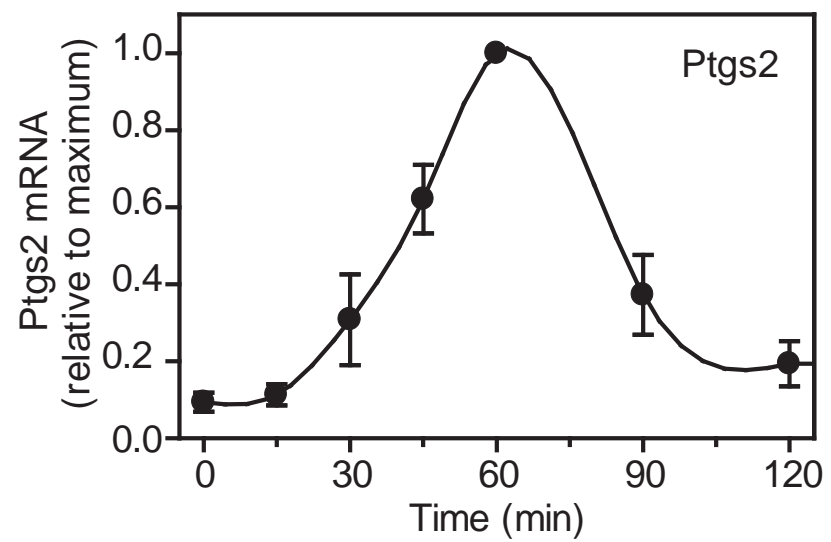

B

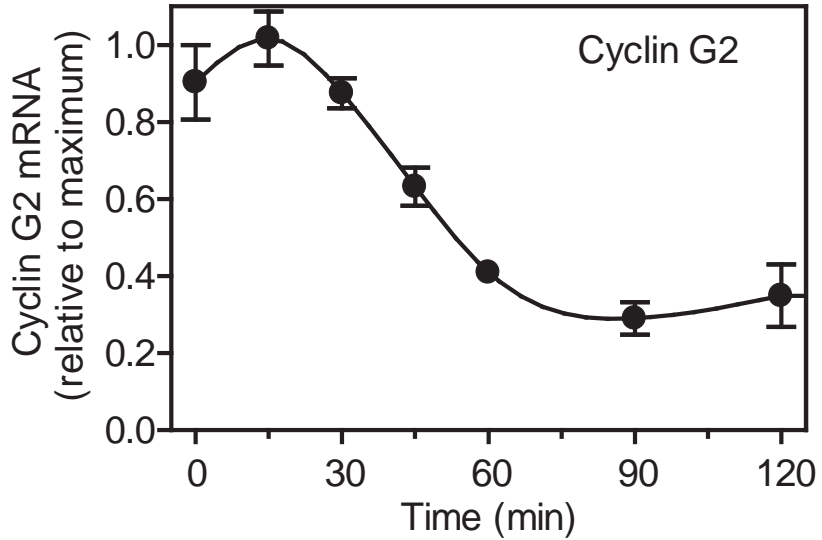

C

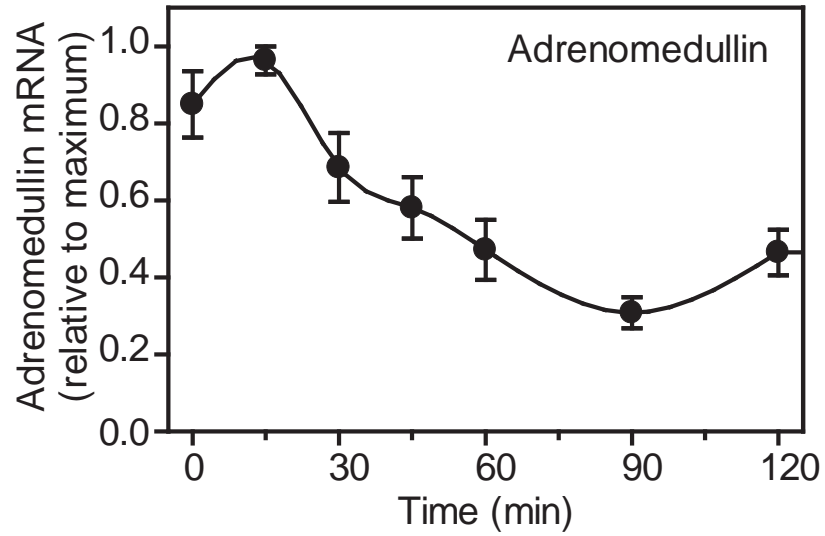

